\title{
Measurement of Various Terms in the Turbulent Kinetic Energy Balance within a Flame and Comparison with Theory
}

\author{
J. F. DRISCOLL and A. GULATI*
}

Department of Aerospace Engineering, The University of Michigan, Ann Arbor, Michigan 48109-2140

\begin{abstract}
Direct measurements have been made of four of the six terms in the exact form of the turbulent kinetic energy (TKE) equation within a premixed turbulent flame, in order to understand how the combustion interacts with the turbulence. Results are compared to the predictions of the Bray-Moss-Libby (B-M-L) model. The mean reaction ( $\bar{w}$ ) also was estimated from the data, using a local control volume concept. The measurements were made possible by the simultaneous use of laser velocimetry and Rayleigh scattering.

Increasing the heat release was found to increase the "flame generated" turbulence as well as the "apparent" turbulence and the turbulent flame speed. Part of this flame-generated turbulence is due to vorticity created by the curved flamelets, as evidenced by the unexpected existence of low speed products. The flame also is found to exert an upstream influence that causes an increase in the turbulence of the pure reactants before these reactants ever reach a flamefront. The measured diffusion term in the TKE balance indicates a countergradient diffusion of TKE toward the center of the flame where TKE is maximum; however, this is not true diffusion since the TKE balance includes apparent turbulence.

The B-M-L model correctly predicts quantities which are dominated by intermittency due to flame motion such as the diffusion term in the TKE, and fluxes $\overline{\rho u^{\prime \prime} c^{\prime \prime}}$ and $\overline{\rho u^{\prime \prime} c^{\prime \prime}}$ which change sign within the flame. This is because the model successfully accounts for intermittency by separating the physics of reactants and products. The model does not successfully predict even the trends associated with the more subtle "true turbulence" associated with products $\left(u_{\mathrm{p}}^{\prime}\right)$. It is felt that additional work is needed to remove "apparent turbulence" from the TKE balance, and eventually to replace one closure relation in the model to account for vorticity produced by curved flamelets.
\end{abstract}

\section{INTRODUCTION}

The question, "How does turbulence enhance the speed of a premixed flame and thus the overall reaction rate?' has been extensively studied, but a number of major questions remain unresolved. Damkohler [1] showed how incident turbulence levels can increase the flame sheet area and thus increase flame speed and overall reaction rate. However, Damkohler's analysis is not complete; Karlovitz [2] and Ballal [3, 4] showed that in addition to the effects of incident turbulence, flame speed can be enhanced by as much as a

\footnotetext{
* Currently employed as Aeronautical Engineer, Staff, General Electric Research Center, Schenectady, New York.
}

factor of ten by flame-generated turbulence. In a ducted flame, vortices generated ahead of the flame can increase flame speed by a factor of 500 [5]. Flame-generated turbulence causes an increase in the turbulence levels associated with the products downstream of the flame front. It is not clear how turbulence downstream of the flame sheet could increase the flame speed; it may increase the flame sheet area directly or it may have an upstream influence and enhance the incident turbulence level. Another reason why Damkohler's analysis is incomplete is that the observed size of flamelet wrinkles may be very different from the scale of the incident turbulent eddies, as discussed below.

It is believed that a logical experimental ap-

Copyright $(1988$ by The Combustion Institute 
proach is first to measure the amount of flamegenerated turbulence at locations within a flame of simple geometry. It is then useful to measure various terms in the exact form of the turbulent kinetic energy balance, in order to understand better why the flame generates turbulence. The present paper discusses the above measurements. The important next step is to use flow visualization to observe how the vorticity field increases the flame sheet area; such efforts are in progress and will be reported in the future.

In the present study it is shown that several important quantities, which cannot be measured using conventional diagnostics, can be measured by the simultaneous use of laser velocimetry and Rayleigh scattering. These diagnostics make possible the measurement of velocity-density correlations, which are useful for the following reasons:

a. The "true turbulence" $\overline{\left(u_{t}^{\prime}\right)^{2}}$, and the "flame generated turbulence" $\overline{\left(u_{\mathrm{fg}}^{\prime}\right)^{2}}$ can be measured after they are separated from the "apparent turbulence" that arises due to flamelet motion over the measurement location. To define true turbulence, it is convenient to consider the reactants and products separately; Bray and Libby [6-9] have shown that

$$
\begin{aligned}
\overline{\rho u^{\prime \prime}} / \bar{\rho}= & (1-\tilde{c}) \overline{\left(u_{\mathrm{r}}^{\prime}\right)^{2}}+\tilde{c} \overline{\left(u_{\mathrm{p}}^{\prime}\right)^{2}} \\
& +\tilde{c}(1-\tilde{c})\left(U_{\mathrm{p}}-U_{\mathrm{r}}\right)^{2},
\end{aligned}
$$

where $\overline{\rho u^{\prime \prime}} / \bar{\rho}$ is the measured Favre-averaged normal velocity fluctuations, $c$ is the measured reactedness as defined below, and $r$ and $p$ denote reactants and products, respectively. The first two terms on the right side of Eq. (1) are denoted "true"' turbulence $\overline{\left(u_{\mathrm{t}}^{\prime}\right)^{2}}$,

$\overline{\left(u_{\mathrm{t}}^{\prime}\right)^{2}} \equiv(1-\tilde{c}) \overline{\left(u_{\mathrm{r}}^{\prime}\right)^{2}}+\tilde{c} \overline{\left(\overline{u_{\mathrm{p}}^{\prime}}\right)^{2}}$,

whereas the last term in Eq. (1) is the apparent turbulence. The flame generated turbulence $\overline{\left(u_{\mathrm{fg}}^{\prime}\right)^{2}}$ thus is

$\overline{\left(u_{\mathrm{fg}}^{\prime}\right)^{2}}=\overline{\left(u_{\mathrm{t}}^{\prime}\right)^{2}}-\overline{\left(u_{0}^{\prime}\right)^{2}}$,

where $\overline{\left(u_{0}^{\prime}\right)^{2}}$ is the initial value of $\overline{\left(u^{\prime}\right)^{2}}$ with no flame present.

b. The mean reaction rate $(\bar{w})$ was estimated from the data using the concept of conservation of species for a control volume:

$$
\bar{w}=d / d x\left(\bar{\rho} \tilde{u} \tilde{c}+\overline{\rho u^{\prime \prime} c^{\prime \prime}}\right) .
$$

Equation (4) is exact since no modeling assumptions are made; it yields only an estimate of $\bar{w}$ since it assumes that gradients which are tangential to the flame are much less than gradients that are normal to the flame, which is shown below to be approximately correct but is not exact in the present experiment. Physically, Eq. (4) states that the mean rate of production of products in a control volume can be measured if one measures the net flux of products that exit through the walls of the control volume. The tilde and double prime represent Favre-averaged mean values and fluctuations, respectively.

c. The correlation measurements allow for a more complete comparison of experimental findings to the Bray-Moss-Libby (B-M-L) model than has been done in the past.

d. Finally, the measurement of velocity-density correlations makes it possible to measure four of the six terms in the exact form of the turbulent kinetic energy balance. The exact form of the TKE balance is now presented for the case of an unconfined flame for which mean flow gradients tangential to the flame are sufficiently less than gradients normal to the flame [7]:

$$
\begin{aligned}
& \bar{\rho} \tilde{U} \frac{d}{d x}\left(\overline{\frac{\rho u^{\prime 2}}{\bar{\rho}}}\right) \\
& 1 \\
& =2(\underbrace{\left.\overline{\frac{-\rho^{\prime} u^{\prime}}{\rho}}\right)\left(\bar{\rho} \tilde{U} \frac{d \tilde{U}}{d x}+\frac{d}{d x} \overline{\rho u^{\prime \prime 2}}\right.}_{2}) \\
& -\overline{2 \rho u^{\prime \prime 2}} \frac{d \tilde{U}}{d x}-\frac{d}{d x} \overline{\left(\rho u^{\prime \prime 3}\right)} \\
& -\frac{}{3}-\frac{d x}{4} \\
& -2 \frac{\overline{u^{\prime \prime} d p^{\prime}}}{d x}-\chi_{\mathrm{u}} \text {. } \\
& 56 \text {. }
\end{aligned}
$$


Term 1 is the convection of the quantity $\overline{\rho u^{\prime \prime 2}} / \bar{\rho}$, which is the component of the TKE associated with the Favre-averaged normal velocity fluctuation $u^{\prime \prime}$. Terms 2 and 3 represent the production and loss of turbulence due to normal stresses; term 3 is the well-known dilatation term which causes a reduction of turbulent kinetic energy per unit volume as a fluid element volume expands in an accelerating flow such as within a flame. Term 2 is a production mechanism by normal stress that arises only in variable density flows, and in fact, only in flows in which $\rho^{\prime}$ and $u^{\prime}$ are correlated. Physically, if a momentum change due to $\rho^{\prime}$ occurs in phase with the fluid motion $u^{\prime}$, work is done in producing velocity fluctuations-that is, work is done only if the effective force is correlated with the fluid motion; if $\rho^{\prime}$ and $u^{\prime}$ are uncorrelated, no net work is done. Bray and Libby described term 2 in another way: the factor $\tilde{\rho} \tilde{U} d \tilde{U} / d x+d / d x \overline{\left(\rho u^{\prime \prime 2}\right)}$ can be shown to be the mean pressure gradient $d \bar{p} /$ $d x$, which preferentially accelerates lower density gas. For example, in an inviscid flow $d p$ $=-\rho U d U$ so lower density parcels experiencing the same pressure difference $d p$ will be given a larger $d U$ than high density parcels, thus leading to velocity fluctuations. Terms 4 , 5 , and 6 represent redistribution of TKE by turbulent diffusion pressure diffusion, and the dissipation of TKE by molecular diffusion, respectively.

One other mechanism that does not appear in Eq. (5) is production of turbulence by shear stresses. In oblique, ducted flames, the velocity vector cannot change direction; thus the gradients $\partial U / \partial y$ and $\partial V / \partial x$ are significant and the shear stress terms must be included in Eq. (5). However, for oblique, unconfined flames, such as the present flame and rod-stabilized flames, $\partial U / \partial y$ and $\partial V / \partial x$ are negligible with respect to $\partial U / \partial x$. Cheng [10] has found that in such flames the true Reynolds shear stresses are negligible.

It is noted that certain terms in Eqns. 1-5 can be measured only if the gas velocity and density are measured simultaneously. Such measurements represent an extension of the authors' previous work in which the effect of heat release on flamegenerated turbulence was described $[11,12]$. Some previous studies have simultaneously utilized LDV and either Mie scattering [13, 14] or thermocouples [15] to demonstrate the existence of countergradient diffusion in flames. Conditional sampling using oil drops also was shown by Cheng [10] to provide a useful assessment of various models. A number of other flame studies have utilized the above diagnostics separately, but not simultaneously [16-20].

\section{EXPERIMENTAL ARRANGEMENT}

\section{A. Turbulent Flame}

A premixed methane-air flame was stabilized on one edge of the rectangular burner shown in Fig. 1. The reactants are passed through two grids: a 30-mesh screen to remove flow nonuniformities, and a 10-mesh turbulence-producing grid. The burner exit is $3.8 \mathrm{~cm} \times 2.54 \mathrm{~cm}$ and is surrounded on all sides by sheath air flow to prevent a shear layer from forming between the primary flow and the stagnant surroundings. The velocities of the primary and sheath flows are matched. The experiment was designed to satisfy three conditions: (i) the flame consists of a single wrinkled flame sheet which has mean flow properties that are as one-dimensional as physically possible and which lies in a uniform field of isotropic gridgenerated turbulence, (ii) the flame brush has a large enough angle of incidence $\theta_{0}$ between the incident flow and the flame to insure that the flame region of interest is several centimeters away from the wake of the stabilizing rim, and (iii) optics were designed to allow for measurements in the directions that are normal and tangential to the flame to allow for direct comparison with the model. It is noted that the present flame is oblique yet unconfined. An oblique, unconfined flame turns the velocity vector since the normal component increases-yet the tangential component $(V)$ remains constant. Confined flames in ducts differ in that the mean velocity vector does not change direction; $V$ increases across the flame and a different model [21] is required to include the effects of Reynolds stresses. The present flame 


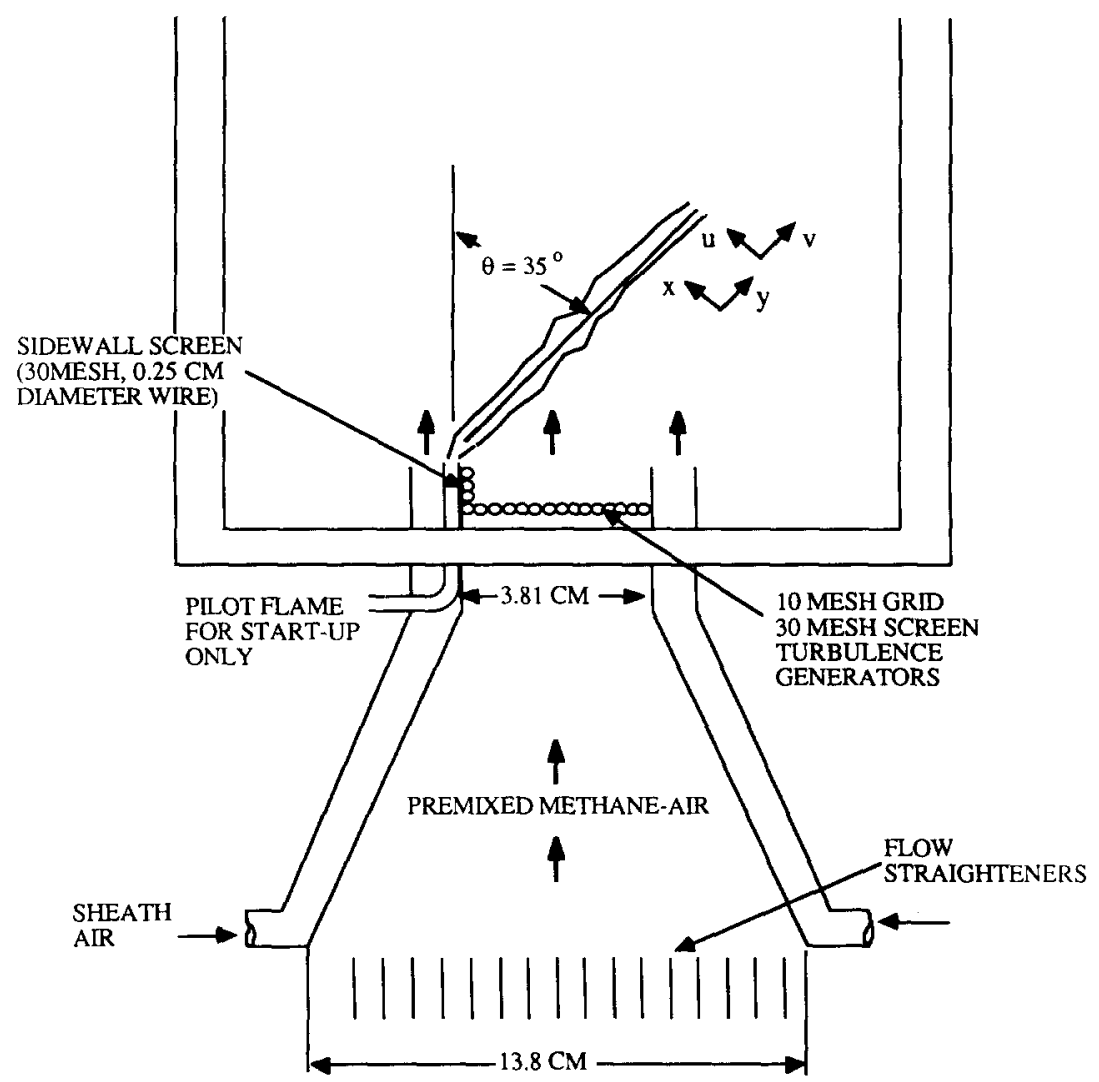

Fig. 1. Schematic of the flame showing the coordinate system.

was surrounded by a $14 \mathrm{~cm} \times 14 \mathrm{~cm} \times 18 \mathrm{~cm}$ enclosure, but since the enclosure width was six times larger than the burner width, it did not confine the flame since $V$ remains constant, as shown below.

The velocity component normal to the flame $(U)$ and tangential to the flame $(V)$ were measured along the $x$-coordinate, which is normal to the flame, as shown in Fig. 1. The origin of the coordinate system is $4.2 \mathrm{~cm}$ above the turbulenceproducing grids. Three flames having equivalence ratios of $0.7,0.8$, and 1.0 were selected for study. As listed in Table 1, the incidence angles $\theta_{0}$ for the three flames were $24^{\circ}, 35^{\circ}$, and $43^{\circ}$, respectively. The heat release parameter $\tau$, defined as $T_{2} / T_{1}$ 1 , where $T$ is the temperature and subscripts 1 and 2 refer to conditions far upstream and downstream of the flame, respectively, was determined from density measurements to be 5.0, 5.5, and 6.4, respectively. Theoretical values of $\tau$ calculated for the known equivalence ratios using the NASA equilibrium chemistry code are given in Table 1.

\section{B. Joint Rayleigh-LV Diagnostics}

The diagnostics used in this study are similar to those developed by Dibble et al. [22] and Driscoll

TABLE 1

List of Various Parameters Associated with the Three Flames

\begin{tabular}{cccc}
\hline Flame & 1 & 2 & 3 \\
\hline$\phi$ & 0.7 & 0.8 & 1.0 \\
$\theta_{0}$ & $24^{\circ}$ & $35^{\circ}$ & $43^{\circ}$ \\
$\tau_{\text {meas }}$ & 5.0 & 5.5 & 6.4 \\
$\tau_{\text {calc }}$ & 5.2 & 5.6 & 6.6 \\
Measured & & & \\
$S_{T}(\mathrm{~m} / \mathrm{s})$ & 0.7 & 1.0 & 1.15 \\
$u_{1}^{\prime} / U_{1}$ & 0.15 & 0.13 & 0.13
\end{tabular}


et al. [23] and are described in detail in Refs. [11] and [12]. The laser velocimeter uses the green beam from a 5-W argon-ion laser and commercial optics. Scattered light is collected in the forward direction using a 50-mm f1.2 camera lens. The signal is sent to a TSI 1980 signal processor. The flow is seeded with alumina particles of $1.0 \mu \mathrm{m}$ nominal diameter using a cyclone seeder.

The Rayleigh system used to measure gas density uses the blue beam from the 5-W argon-ion laser, which is focused to a waist diameter of 100 $\mu \mathrm{m}$ using the same lens that is used for velocity measurements. The length of the optical volume in the laser beam direction is $1 \mathrm{~mm}$. The Rayleigh scattered light is collected at $90^{\circ}$ using an $85-\mathrm{mm}$ f 1.2 camera lens, an RCA 4526 phototube, a 1.5 $\mathrm{mm} \times 150 \mu \mathrm{m}$ slit, and a $0.3 \mathrm{~nm}$ bandpass filter. The signal is filtered, amplified, and digitized with a 14-bit TECMAR A/D converter. The RC time constant of the system is $100 \mu \mathrm{s}$. Background light and flame luminescence are less than $1 \%$ and $5 \%$ of the signal, respectively. All the optics are rigidly mounted and the burner is traversed.

Exact simultaneity between density and velocity measurements is not possible since the light scattered from the particles that are required for velocity measurements renders the Rayleigh signal invalid. In this study density was measured, on the average, $120 \mu$ s prior to velocity measurements. The $120 \mu$ s nonsimultaneity time previously has been shown to be sufficiently small for accurate measurements [12], as described below. To avoid multiple particles in the probe volume, the seeding rate was carefully monitored to keep the velocity validation rate below 10 samples per second. At every location, 2048 density-velocity pairs were stored in a computer.

The joint density-velocity measurements are slightly biased because the mass flux $\rho U$ is not constant but decreases by $35 \%$ across the flame, as discussed below. Hence, all experimental results have been corrected for bias in the following manner:

$$
\begin{gathered}
U=\frac{\sum\left(1 / \rho_{i}\right)}{\sum\left(1 / \rho_{i} U_{i}\right)}, \quad \bar{\rho}=\frac{\sum\left(1 / U_{i}\right)}{\sum\left(1 / \rho_{i} U_{i}\right)}, \\
\overline{\left(\rho^{\prime} u^{\prime}\right)}=\frac{\sum\left(\left(U_{i}-\bar{U}\right)\left(\rho_{i}-\bar{\rho}\right) / \rho_{i} U_{i}\right)}{\sum\left(1 / \rho_{i} U_{i}\right)},
\end{gathered}
$$

etc.
This type of bias correction has been proposed by McLaughlin and Tiederman [24]. The bias corrected values were found to agree with the correct time average values of velocity and density to within 5\% throughout the flame [11]. The maximum bias correction was $15 \%, 30 \%$, and $20 \%$ for $U, \bar{\rho}$, and $\overline{\rho^{\prime} u^{\prime}}$, respectively.

The reactedness $c$ was deduced from the Rayleigh scattering data using the definition of $c$, which is $\left(\rho_{1} / \rho-1\right) / \tau$, where $\tau$ is $\left(T_{2} / T_{1}-1\right)$ and subscripts 1 and 2 denote conditions far upstream and downstream of the flame. Since the gas density PDF has two very distinct peaks [11], the data could be separated into reactants and products, and the mean velocity of reactants $\left(U_{\mathrm{r}}\right)$, for example, was deduced by ensemble-averaging the velocity of all samples identified as reactants.

The two major sources of experimental error were shot noise and a finite sample size. Shot noise was determined to be less than $5 \%$ of the gas density signal by measuring the rms fluctuations in a constant density gas. Shot noise affects $\rho_{\mathrm{rms}}^{\prime}$ but does not contribute to velocity-density correlations or $\bar{\rho}$ since it is random in nature. Error due to finite sample size (2048 samples per location) was determined by calculating the deviation between data points and a best fit curve. The scatter was $12 \%$ for terms in the TKE equation but only $2 \%$ for rms fluctuations in velocity and density. This error can be halved by quadrupling the number of samples [25].

In order to compare the experiment measurements with the predictions fo the B-M-L model, a numerical code was written to solve the two governing ordinary differential equations of the B$\mathrm{M}-\mathrm{L}$ model. All of the terms and coefficients were exactly the same as that of Ref. [7]. The two input parameters that characterize the present experiment were the initial turbulence level of reactants $\left.\overline{\left(u_{1}^{\prime 2} /\right.} U_{1}^{2}\right)$ and $\tau$, the heat release parameter. Both parameters were set equal to the values measured in the present experiment and are listed in Table 1. To check the solution algorithm, the equations were solved for the original input parameters used by Bray et al. [7] and the results of Ref. [7] were duplicated.

It is noted that the experimental results are plotted using the independent variable $\tilde{\boldsymbol{c}}$, which is the Favre-averaged reactedness. This is done in 
order to allow for comparison with theory. The model requires that the independent variable $\tilde{c}$ be used; to obtain solutions in terms of the spatial variable $x$, a submodel for the mean reaction rate $\bar{w}$ would be required. A submodel for $\bar{w}$ has been proposed by Bray et al. [26]. However, because of the preliminary nature of such a submodel, it was decided not to use any submodel for $\bar{w}$ in comparing theory to experiment.

\section{RESULTS}

\section{A. Flame Structure}

The flame, as shown in Figs. 1 and 2, appears to be very turbulent in that the brush thickness is greater than $1 \mathrm{~cm}$ and the flame speed is four times that of a laminar flame of corresponding stoichiometry. The structure of the flame is shown in
Fig. 2 to consist of very thin wrinkled flamelets that are semicircular and cusped toward the product side. The cusped wrinkles are the remnants of a laminar flame instability that persists in turbulent laboratory flames [27]; by raising the velocity in the present study by a factor of five, the cusped structure was still observed. The instabilities can be either thermodiffusive or hydrodynamic in nature [1, 27-30].

Figure 2 shows that the $1-\mathrm{cm}$ size of the wrinkles does not equal the integral scale of the incident turbulence, which is less than $0.1 \mathrm{~cm}$ in the present flame [11]. Schlieren movies of the present flame [12] and other flames [27] show that each curved wrinkle is produced near the stabilization point, presumably by the incident turbulence, and the wrinkle acts like a transverse wave that propagates along the flame sheet at approximately the tangential velocity $(V)$. Damkohler's theory
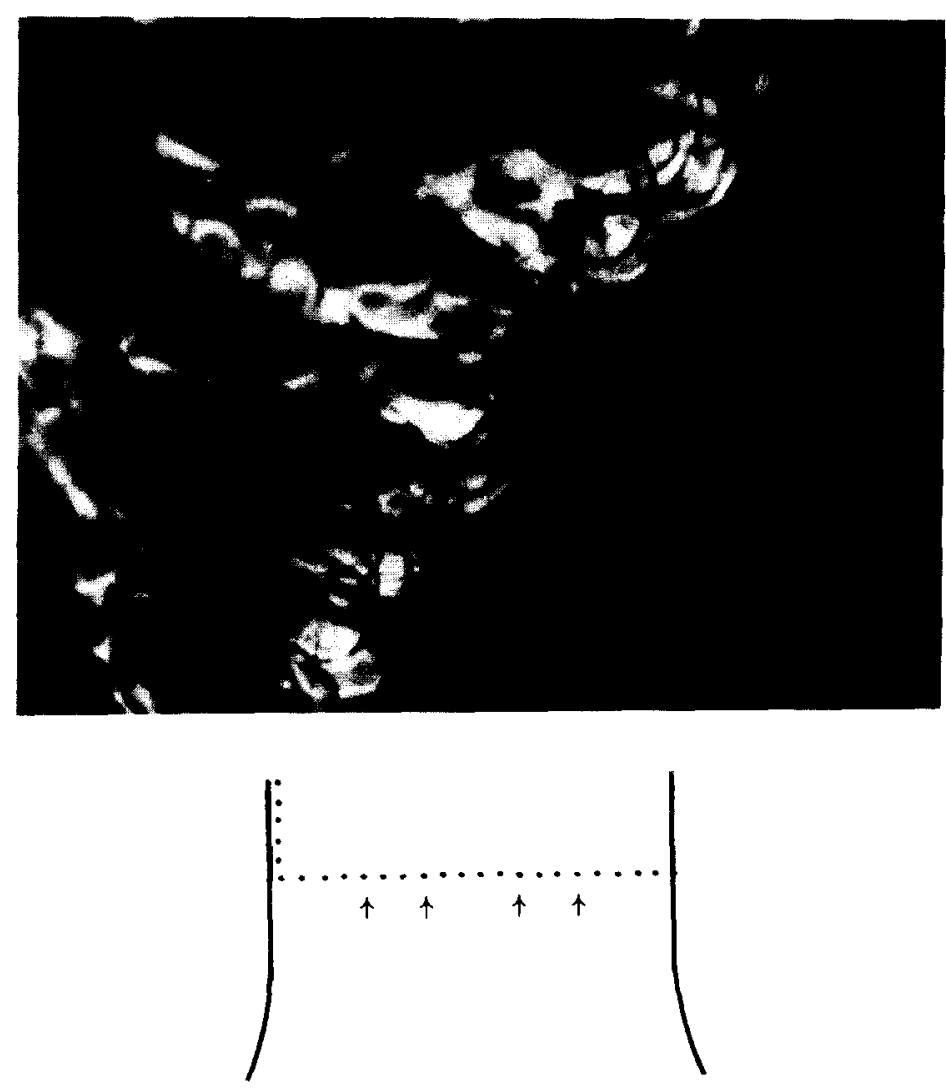

Fig. 2. Schlieren photograph of the flame showing curved wrinkled flamelets. $\phi=0.8$, turbulent flame speed $S_{T}=1.0 \mathrm{~m} / \mathrm{s}$, corresponding laminar flame speed $=0.25 \mathrm{~m} / \mathrm{s}$. 
of turbulent flame speed [1] assumes that wrinkle size corresponds to the size of eddies in the incident flow, which does not appear to be the case in the present flame. The flame sheet area, however, does appear to increase by more than a factor of three because of the wrinkles, and Damkohler's theory explains how an increase in flame sheet area causes an increase in flame speed.

Precautions were taken to make the present flame as one-dimensional in mean properties as possible, and in direct photographs the brush has the appearance of a $1-\mathrm{cm}$ thick planar sheet. However some deviation from one-dimensionality is observed in the mean profiles of normal velocity and density, shown in Fig. 3. The mass flux $\bar{\rho} \tilde{U}$ downstream of the flame was $35 \%$ less than $\tilde{\rho} \tilde{U}$ upstream of the flame; this type of deviation from one-dimensionality is commonly observed in other flames and can be attributed to the observation that flame brush thickness increases as the distance from the stabilizing rod or rim increases [10]. As the heat release $\tau$ increases, Fig. 3 shows that downstream values of $\bar{\rho}$ and $\tilde{U}$ decrease and increase, respectively, as expected. The profiles in Fig. 3 display the expected trends at upstream and downstream locations; however, the density profile for $\tau=5.5$ (solid triangles) is shifted to the left of other density profiles because the $\tau=5.5$ flame happened to stabilize at a position slightly upstream of the other two flames.

\section{B. Flame-Generated Turbulence}

It has been recognized that velocity fluctuations in a flame are the sum of two components: "true" flame turbulence $\overline{u_{\mathrm{t}}^{\prime 2}}$ associated with the reactants and the products, as well as the "apparent turbulence" associated with the intermittent passing of flamelets over the measuring point. Equations (1)-(3) allow the two components to be separated. Measured values of $\overline{u_{t}^{\prime 2}}$ are shown in Fig. 4. The flame-generated turbulence in Fig. 4 is observed to increase monotonically across the flame. Therefore the reason that the total velocity fluctuation reaches a local maximum in Fig. 4 is entirely due to the velocity PDF evolving from Gaussian form to a bimodal form and then back to a Gaussian form across the flame. In the past, the decrease in velocity fluctuations has sometimes incorrectly been attributed to dilatation.

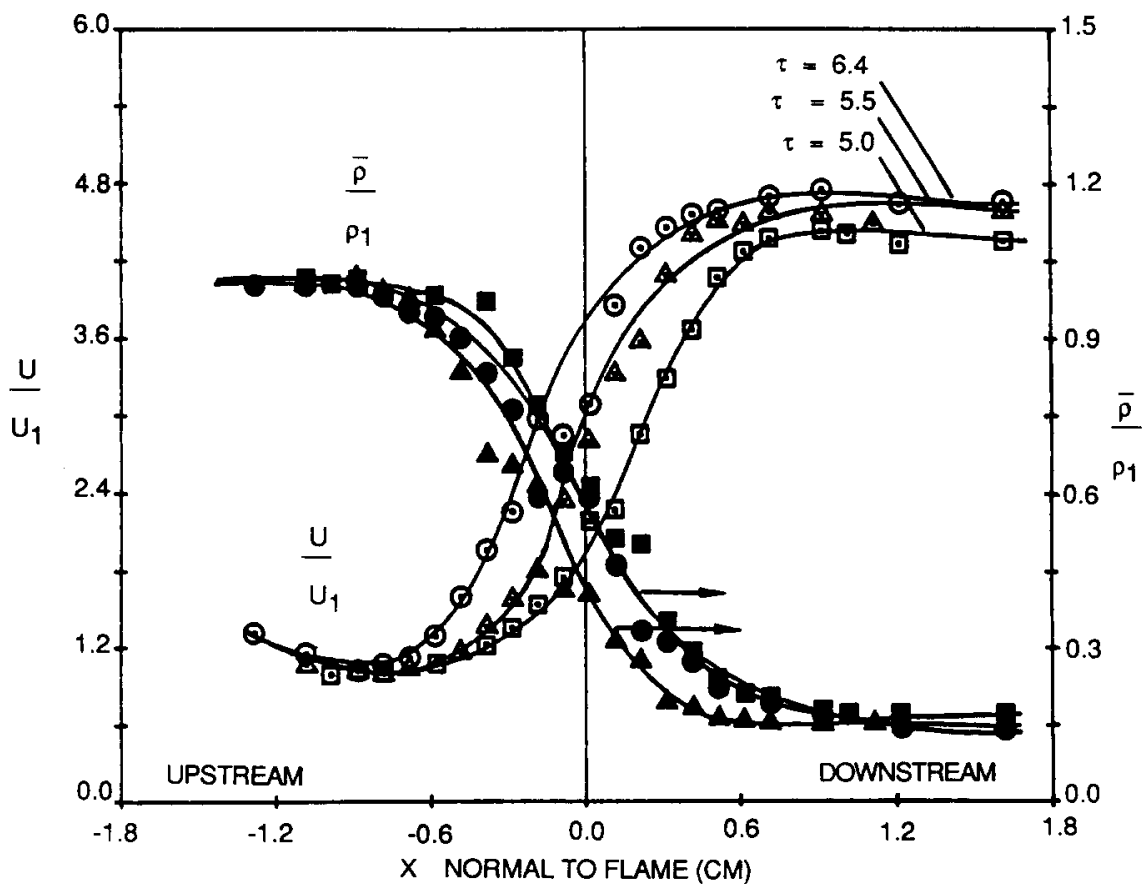

Fig. 3. Mean normal velocity and gas density for various heat releases. 


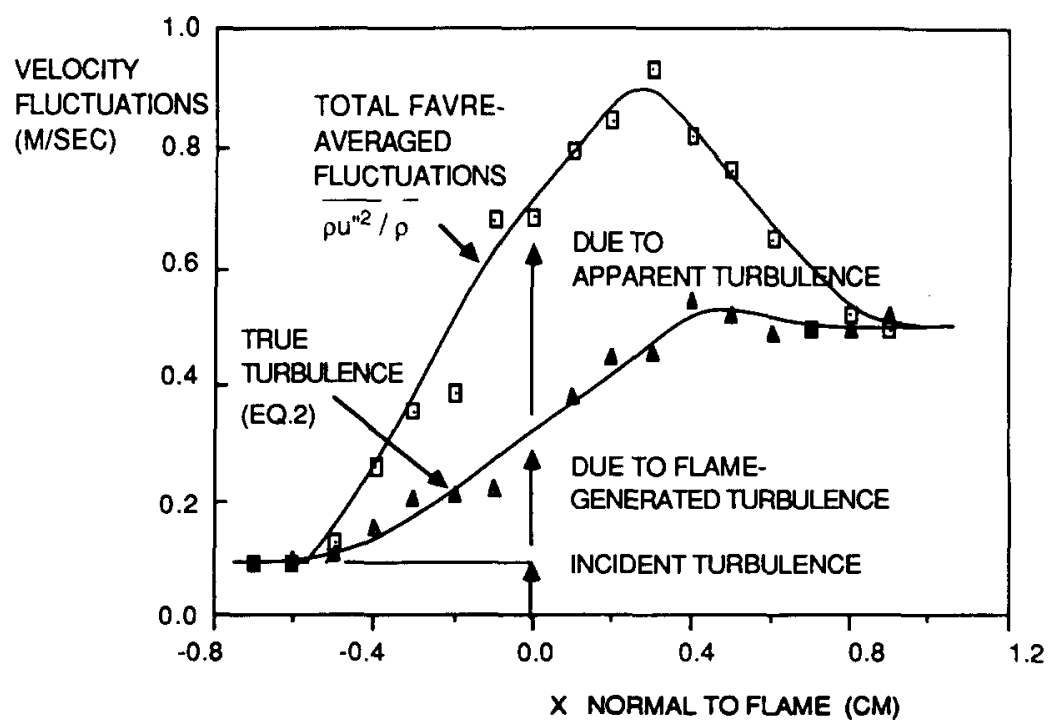

Fig. 4. Measured flame-generated turbulence as defined in Eqs. (1)-(3). $\tau=5.5$.

The effect of heat release on the Favre-average velocity fluctuations is shown in Fig. 5. For comparison, the values predicted by the B-M-L model also are shown. Of particular interest is the ratio $u_{2}^{\prime} / u_{1}^{\prime}$. This ratio represents the total amount of turbulence imparted by flame to the flow. Values of $u_{2}^{\prime} / u_{1}^{\prime}$ were $2.1,4.2$, and 5.3 for values of heat release $(\tau)$ of $5.0,5.5$, and 6.4 , respectively. Thus, in all cases studied, the flamegenerated turbulence since $u_{2}^{\prime} / u_{1}^{\prime}$ exceeds unity, and the amount of flame-generated turbulence increases with heat release, as predicted by the B$\mathrm{M}-\mathrm{L}$ model results in Fig. 5.

One important result of Fig. 5 is that both

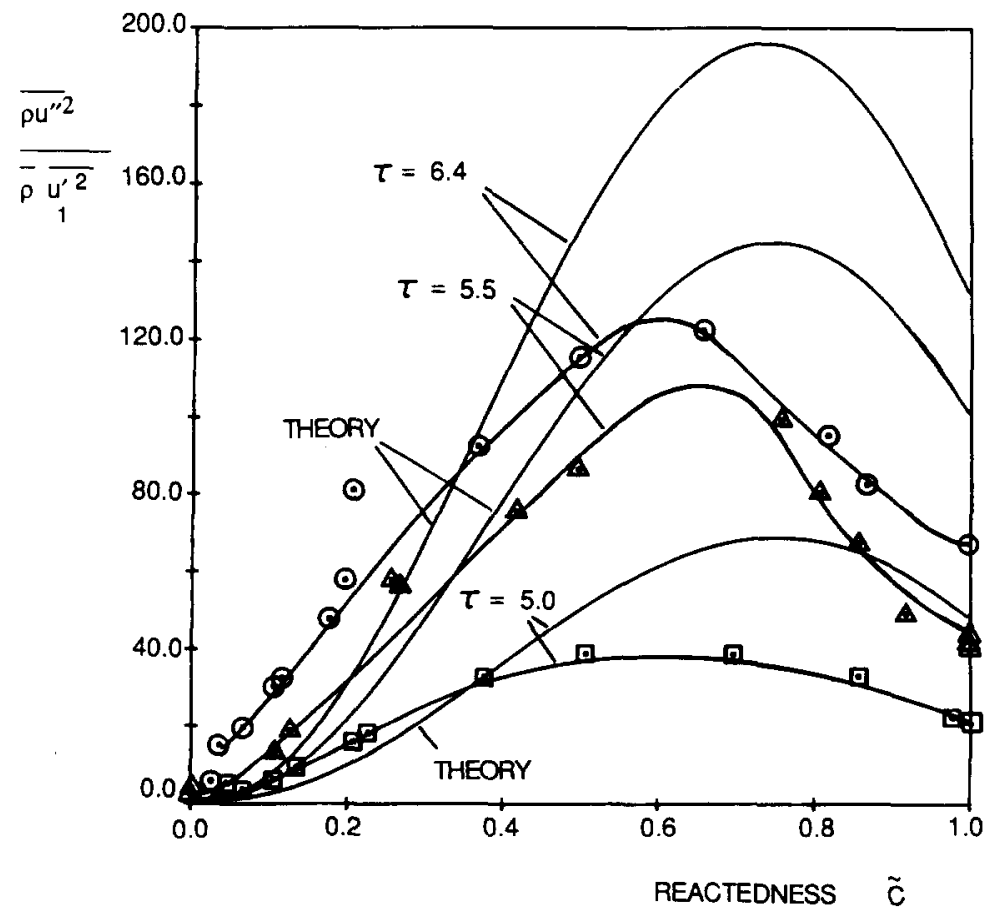

Fig. 5. Favre-averaged normal velocity fluctuations compared with B-M-L model. 
theory and experiment show that increasing the incident turbulence level actually decreases the relative rise in velocity fluctuations in the flame. That is, Fig. 5 shows that for relatively low incident turbulence $\left(u_{1}^{\prime} / U_{1}=13 \%\right)$ the velocity fluctuations rise by a factor of 11 , which is approximately the square root of the maximum measured quantity in Fig. 5, $\tau=6.4$. However, Moss [7] used a much larger incident turbulence level of $40 \%$ and saw only a fivefold increase in velocity fluctuations (and $\overline{\rho u^{\prime \prime 2}}$ ). The B-M-L model corrected predicts this trend, as is seen by comparing the predictions in Fig. 5 for low incident turbulence and the predictions of Bray [7, Fig. 2a] for high incident turbulence. Apparently, as incident turbulence level $\left(u_{1}^{\prime}\right)$ increases, the absolute velocity fluctuations caused by flame motion do not increase; thus the ratio $u^{\prime} / u_{1}^{\prime}$ must decrease.

The magnitude of velocity fluctuations predicted by the B-M-L model in Fig. 5 does not agree with the present data, although the trends are in agreement. The disagreement exceeds any experimental uncertainty and instead is believed to be due to the nonuniversal nature of certain empirical closure relations in the theory such as Eq. (10), which will be discussed later. Until the physics of turbulence generation are better understood and certain empirical relations can be replaced, a general method to predict the magnitude of turbulence in a flame, and eventually the turbulent flame speed, may not be possible.

\section{Turbulent Kinetic Energy (TKE) Balance}

Each of the first four terms in the TKE balance given by Eq. (5) was measured and compared to the B-M-L model predictions. Some results are shown in Fig. 6. The convection term, which is labeled term 1 in Eq. (5), is proportional to the gradient of velocity fluctuations; thus if the sum of terms $2-6$ is positive, velocity fluctuations will increase in the $x$ direction. Figure 6 shows that the convection term 1 changes sign, which is expected, since Fig. 5 had shown that the velocity fluctuations $\rho u^{\prime \prime 2} / \rho$ first increase and then decrease in the $x$ direction.

It should be remembered that velocity fluctations $\overline{\rho u^{\prime \prime}} / \bar{\rho}$ are comprised of both "true turbu- lence" and "apparent turbulence," as was shown by Eqs. (1)-(3). A more revealing equations arises when Eqs. (1)-(3) are substituted into Eq. (5), leading to terms such as $d / d x \overline{\left(u_{\mathrm{fg}}^{\prime}\right)^{2}}$ which indicate how the true flame-generated turbulence should increase across a flame. Because of the resulting complexity, further work is needed before definite conclusions can be drawn from such an equation.

Figure 6 shows that the production rate [term 2 in Eq. (5)] dominates over the dilatation loss (term 3 ) in the upstream portion of the flame, yet the reverse is true in the downstream portion. Part of this trend is explained by the apparent turbulence. The apparent turbulence contributes to the production term since the $\overline{\rho^{\prime} u^{\prime}}$ correlation factor in the production term is almost entirely due to flamelet motion over a point. Similarly, apparent turbulence contributes to the dilatation term 3 in Eq. (5) because $u^{\prime \prime}{ }^{2}$ includes a contribution from apparent turbulence as shown by Eq. (1). Thus the production term increases rapidly in the upstream region partly because apparent turbulence increases as the velocity PDF evolves from Gaussian to bimodal form.

The rate of diffusion of turbulence is shown by the data in Fig. 6 to follow a countergradient trend. That is, $\overline{u^{\prime \prime 2}}$ reaches a maximum near the center of the flame at $\tilde{c}=0.6$ in Fig. 5, yet there is diffusion of $\overline{u^{\prime \prime 2}}$ toward $\tilde{c}=0.6$ since the diffusion term in Fig. 6 indicates a net gain at $\tilde{c}=$ 0.6 . Figure 7 more clearly illustrates the countergradient diffusion of TKE; the flux of $\overline{u^{\prime \prime 2}}$ in the $x$ direction is given by $\overline{\rho u^{\prime \prime} /} / \rho_{1} U_{1}{ }^{3}$, which is shown to be positive in the upstream portion and negative in the downstream portion of the flame. While the magnitude of the measured flux of TKE in Fig. 7 does not agree with the predicted flux (also shown in Fig. 7), the agreement in sign reversal is an encouraging aspect of the model. Also, the fact that the maximum predicted flux in Fig. 7 increases with heat release is in agreement with experiment.

\section{Additional Turbulence Generation Mechanisms}

Conditional sampling measurements are especially useful because they show that additional mecha- 

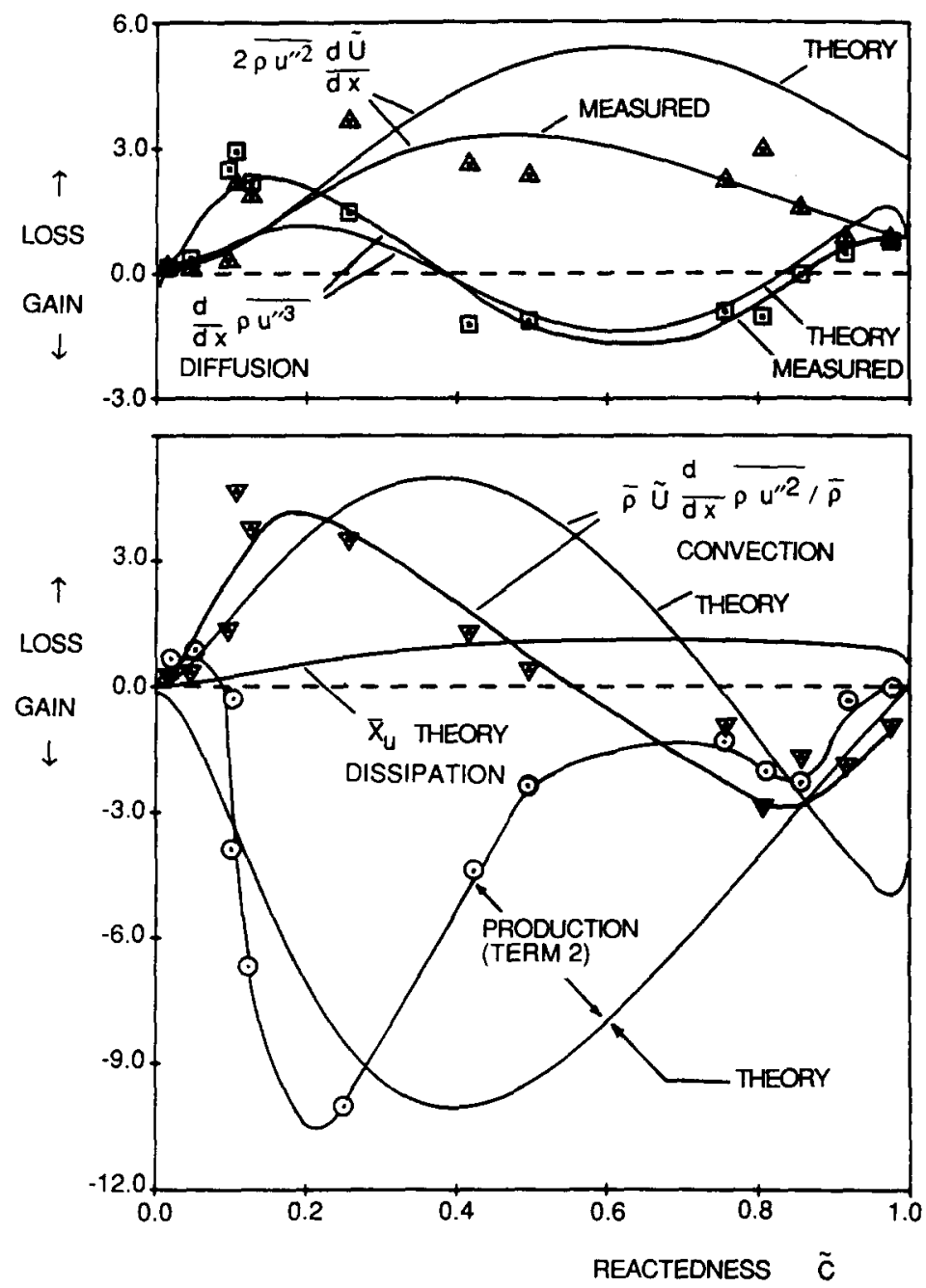

Fig. 6. Measured terms in the turbulent kinetic energy equation [Eq. (5)] across the flame ( 7 $=5.5)$. All quantities are normalized by $\rho_{1} U_{1}{ }^{3}(d \tilde{c} / d x)$.

nisms, which are not explicitly included in the B$\mathrm{M}-\mathrm{L}$ model, also must be important in the present flame. Two such mechanisms are (1) the production of vorticity by curved flamelets and (2) creation of turbulence in the reactant gas parcels before these parcels reach the reaction zone. Figure 8 shows that the velocity PDF associated wit reactants remains Gaussian throughout the flame. However, the velocity PDF of products is not Gaussian, which contradicts one of the assumptions of the B-M-L model. At the flame center, Fig. 8 shows that about $75 \%$ of the products travel at relatively high velocity, as expected, but about $25 \%$ of the products remain at low velocity; i.e. they apparently are not accelerated by the flamelets. The presence of both high and low speed products broadens the PDF (the PDF in Fig. 8 at $x=1.2 \mathrm{~cm}$ is much wider than at $x=-1.2 \mathrm{~cm}$ ) and thus results in flame-generated turbulence.

It is postulated that the observed low velocity products are a result of the curved flamelets that were shown in Fig. 2. Figure 9 shows that gas which crosses a region of the flamelet that is perpendicular to the $x$ direction experiences a sixfold increase in $U$, yet gas that crosses a region of the flamelet that is nearly tangential to the $x$ direction experiences no increase in $U$ and be- 


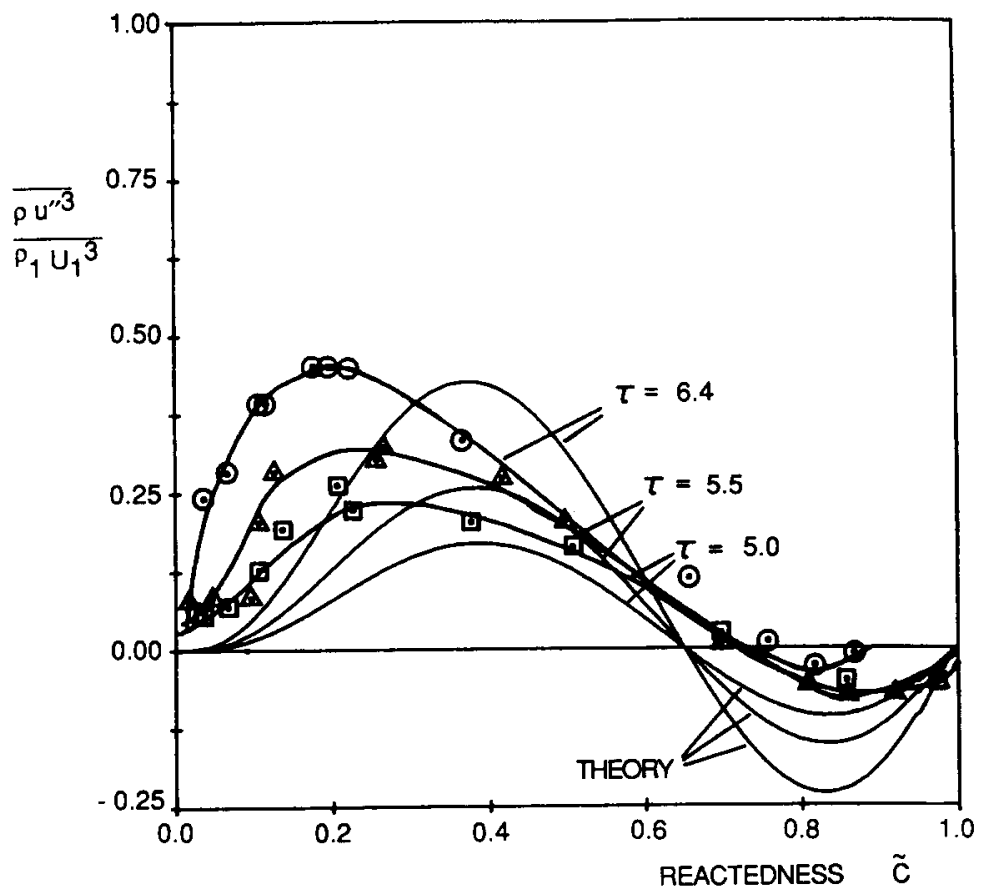

Fig. 7. Flux of turbulent kinetic energy compared with B-M-L model predictions.

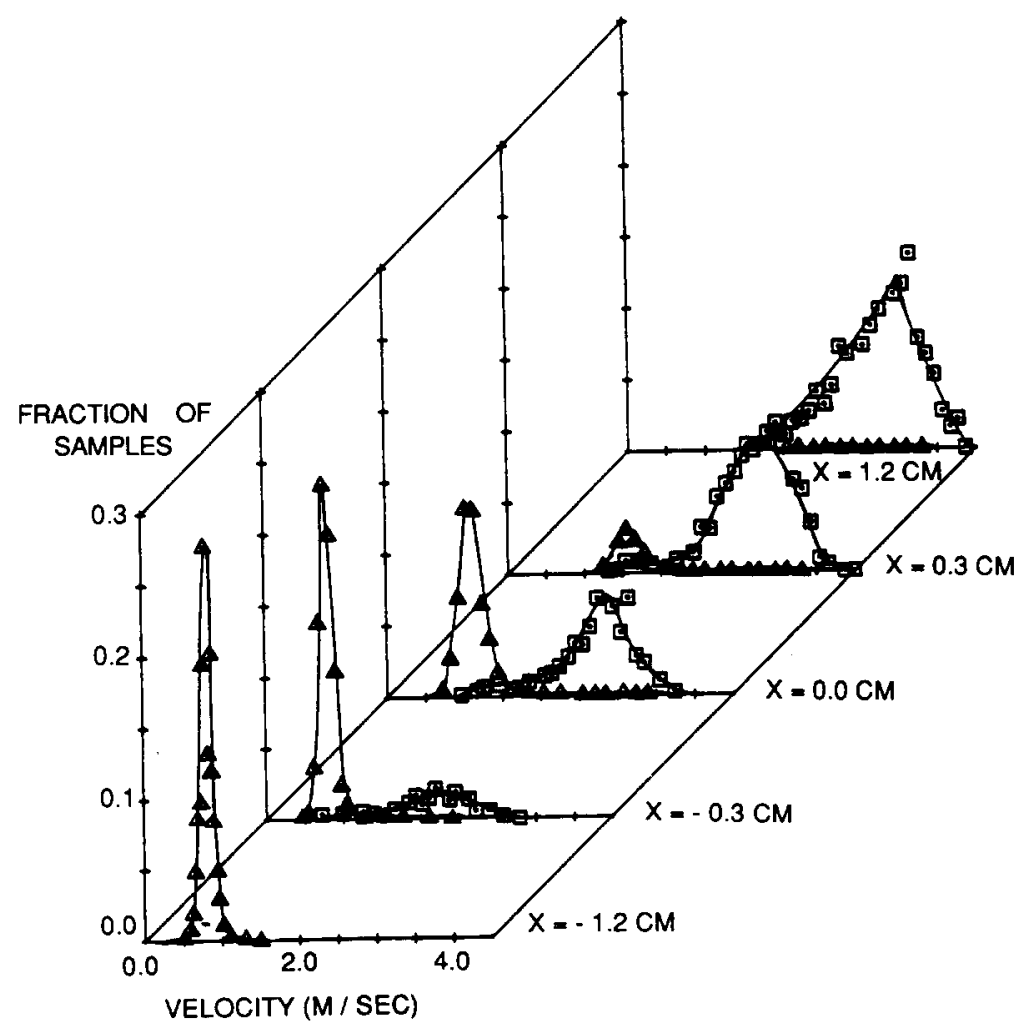

Fig. 8. Evolution of conditioned normal velocity PDFs of reactants and products: $(\Delta)$, reactants; $(\square)$, products. $\tau=5.5$. 


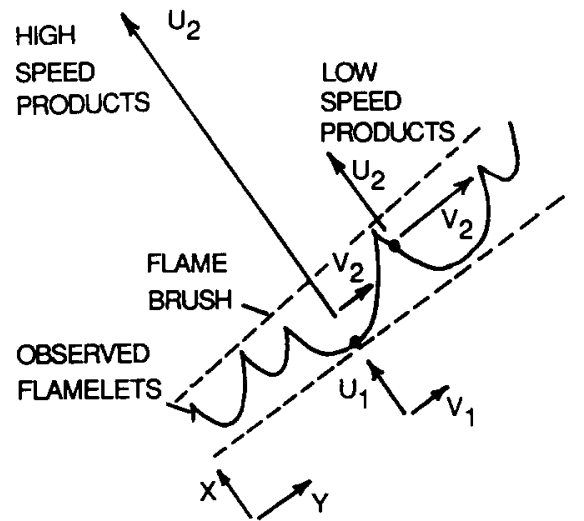

Fig. 9. Schematic of how both the low speed products and the high speed products of Fig. 8 can form due to curved flamelets, leading to vorticity fluctuations.

comes a "low velocity product." This latter gas parcel should experience an increase in $V$; thus the mechanism should cause both $u^{\prime}$ and $v^{\prime}$ to increase across the flame, and by symmetry arguments, no increase in $V$ is expected. In fact, Fig. 10 shows that $v^{\prime}$ does increase across the present flame, but $V$ remains nearly constant, which further substantiates the above mechanism. The authors previously concluded that the observed curved flamelets caused the observed velocity differences in the products $[11,12]$ and later realized that their explanation is the same as that postulated by Karlovitz [2] and described by Lewis [1], Section VI.3. Therefore, the present results provide direct experimental evidence that the Karlovitz mechanism exists; such evidence had been lacking to date. Emmons [32] also describes how curved flames are similar to curved shock waves in that they produce vorticity. Erroneous skewing of the product velocity PDF due to possible passage of the flamelet during the 120 $\mu \mathrm{sec}$ nonsimultaneity time (discussed earlier) was concluded to be negligible; the flamelet crossing frequency was only $250 \mathrm{~Hz}$, thus only one sample out of 33 samples could be affected; also, the skewing of the reactant velocity PDF would be equally likely but was not observed.

The measurements also indicate that the flame creates turbulence within the reactants and alters the mean velocity of reactants before they reach the flame. One could envision the hypothetical case in which all flamelets are parallel and onedimensional and velocity change occurs only at the flamelet interface. For such a case, the values of $U_{\mathrm{r}}$ and $U_{\mathrm{p}}$ would be constant in the $x$ direction and $U_{\mathrm{r}}$ would equal $U_{1}$ while $U_{\mathrm{p}}$ would equal $U_{2}$. Instead, the measurements of Fig. 11 show that $U_{\mathrm{r}}$ and $U_{\mathrm{p}}$ increase in the $x$ direction. This is believed to be due to the mean pressure gradient; each flamelet contributes a small instantaneous pressure drop and the resulting favorable mean pressure gradient accelerates both reactants and products everywhere. The increase in $U_{\mathrm{r}}$ in Fig. 11 represents an "upstream influence" due to the pressure

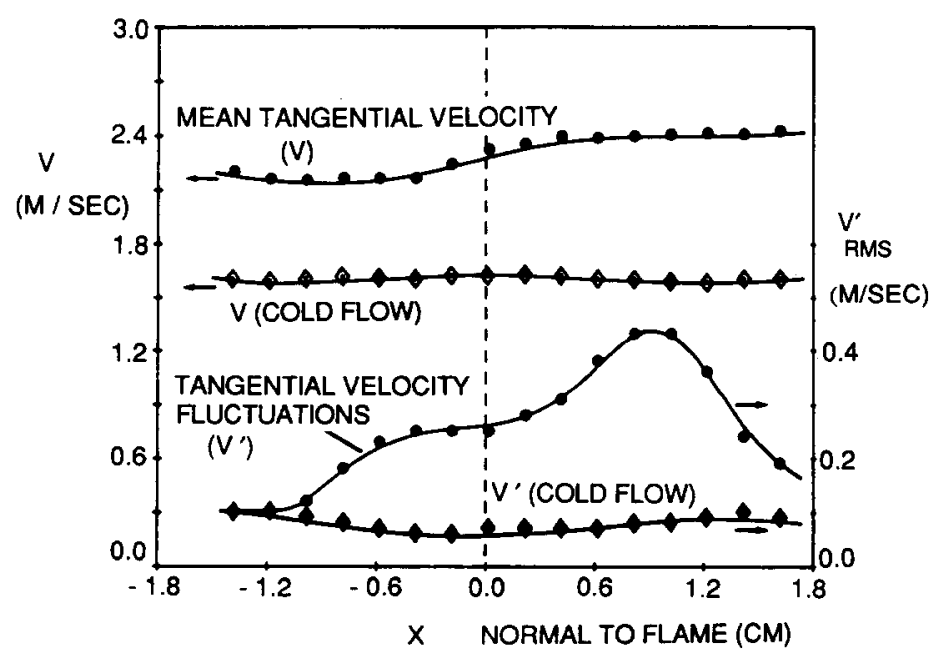

Fig. 10. Measured increase in tangential velocity rms fluctuations due to flame. 


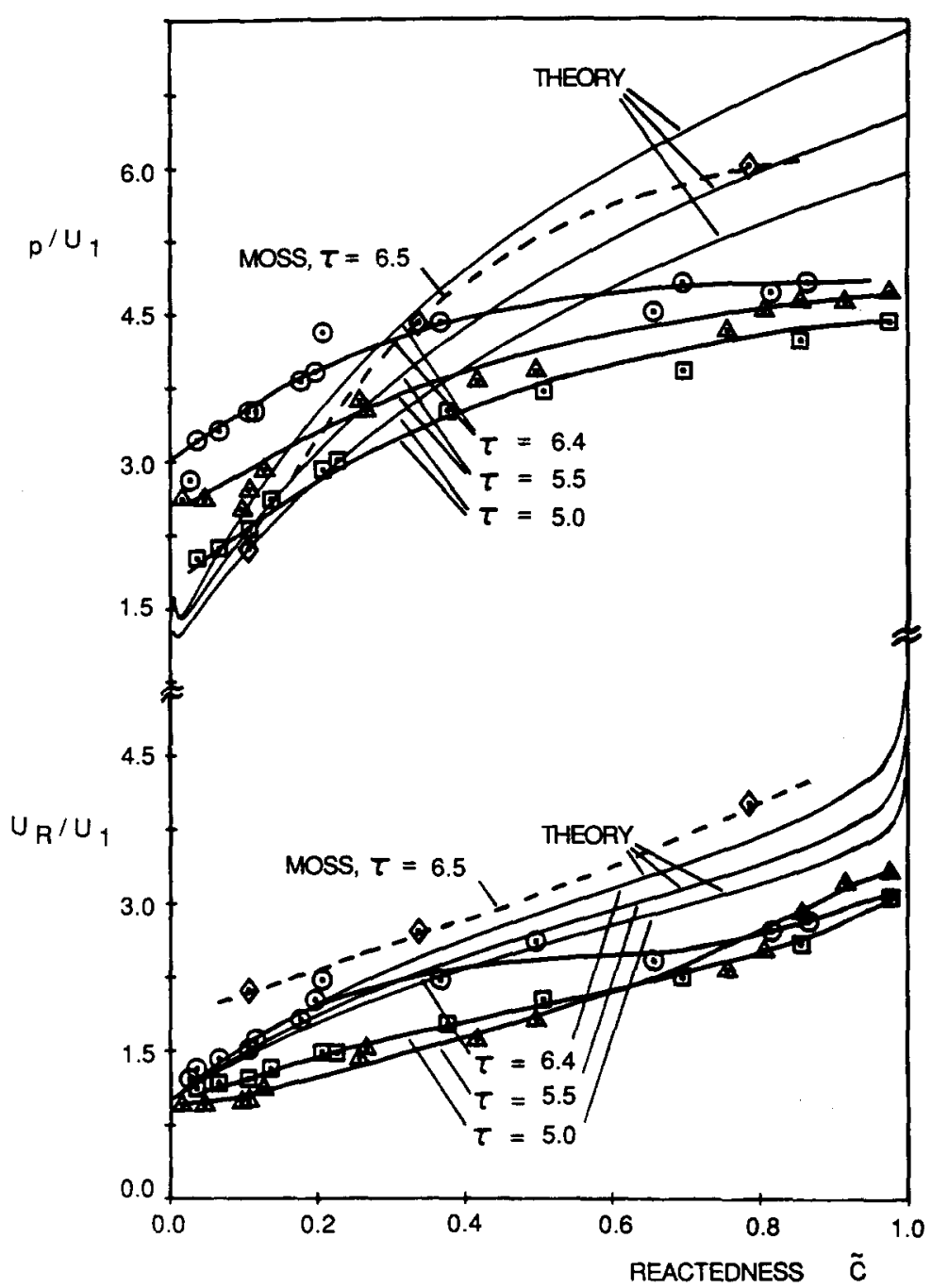

Fig. 11. Effect of heat release on mean conditioned velocities of products and reactants. Data of Moss [13] also are shown.

field generated by the flamelets; that is, the reactant gas is found to be accelerated before it arrives at a flamelet. Cheng [10] also reports that $U_{\mathrm{r}}$ increases across his flame. Figure 11 also shows that the measurements and the B-M-L theory agree that increased heat release increases $U_{\mathrm{r}}$ and $U_{\mathrm{p}}$.

A similar "upstream influence" of the flame on the turbulence associated with reactants $u_{\mathrm{r}}^{\prime}$ is found, as shown in Fig. 12. Gas parcels which have not yet arrived at a flamelet are found to experience an increase in turbulence as they flow in the $x$ direction. Previous results by Shepherd and Moss [14] also show that $u_{r}^{\prime}$ increases in the $x$ direction. The B-M-L theory also predicts an increase in $u_{\mathrm{r}}^{\prime}$, as shown in Fig. 12, but the physical reasons are not apparent because an empirical relation between $u_{\mathrm{p}}^{\prime}, u_{\mathrm{r}}^{\prime}$, and mean quantities is employed in the theory. Three possible mechanisms can explain how the flame adds turbulence to the upstream reactants. (a) The unsteady pressure field can affect $u_{\mathrm{R}}^{\prime}$; as a gas parcel burns, it expands rapidly and increases its specific volume by a factor of six; the velocity 


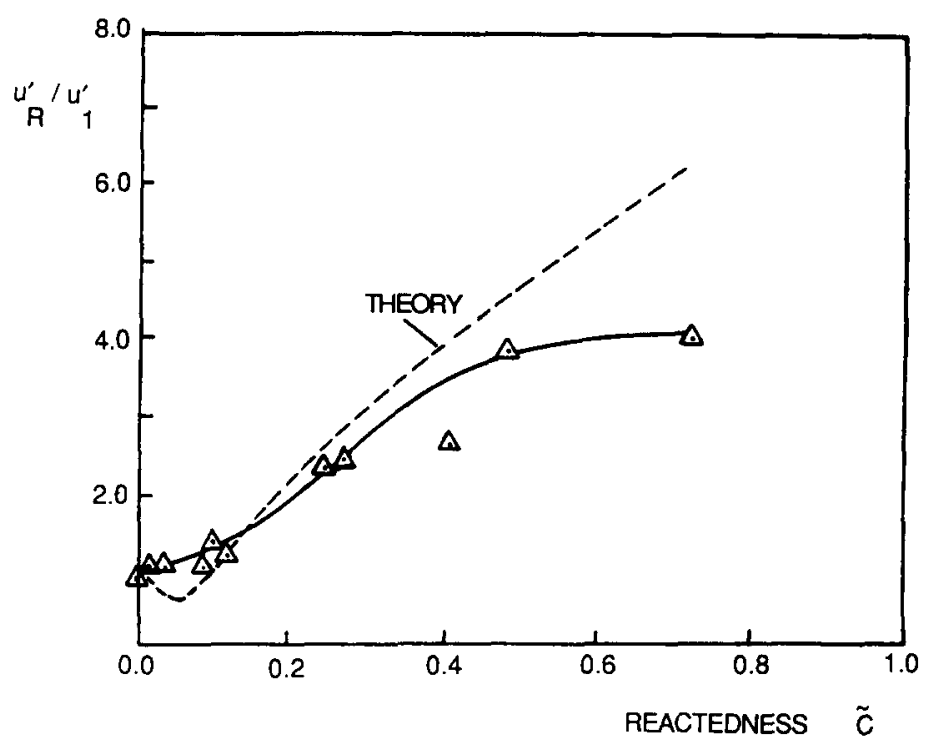

Fig. 12. Turbulence level associated with reactants in the flame $(\tau=5.5)$.

field upstream of a rapidly expanding sphere would vary in time as a streamline deflection occurs due to the pressure field. The $\overline{u^{\prime} p^{\prime}}$ term in the TKE equation is neglected in the B-M-L model but it may be important. While the instantaneous pressure fluctuation associated with a flamelet passage is small, it is sufficiently large to cause as much as a sixfold increase in velocity across that flamelet. (b) Vortices, created by curved flamelets described previously, are added to the product gas and will affect the gas upstream of the flame, according to the Biot-Savart law. A promising method to model the flame-vortex interaction is being developed by Ashurst [33]. (c) Flamelet wrinkles can distort the streamlines upstream of the wrinkle; this effect is observed in laminar flames in tubes [34; 1 , Section V.9]. A deflection of the upstream flow also was observed by Cheng [31, Fig. 3]. Gas expansion causes the flame to act as a bluff body and the flow upstream of the leading edge of the curved flame is decelerated just as flow upstream of the stagnation point of a bluff body is decelerated, which, due to the unsteady appearance of wrinkles, can lead to increases in $u_{\mathrm{R}}^{\prime}$.

Flow deceleration ahead of each wrinkle also may explain how flamelets propagate. In Fig. 2, the reactants approach the flame brush in the $x$ direction (defined in Fig. 1) at four times the laminar flame speed, yet portions of the flamelets remain normal to the $x$ direction. Either those portions of the flamelet propagate at four times the laminar flame speed due to small scale turbulence or, as is more likely, the incident flow is deflected as with a curved laminar flame within a tube.

\section{E. Mean Reaction Rate}

The mean reaction rate $(\bar{w})$ was estimated from the data using a control volume approach. By definition, $\bar{w}$ is the average mass of products that is formed in a control volume, per second per unit volume. Values of $\bar{w}$ were estimated using the species conservation equation given by Eq. (4); this equation simply equates the rate of products formed in a control volume to the difference between the rates of products leaving and entering that control volume. Results that are shown in Fig. 13 are only estimates because $\bar{\rho} \tilde{U}$ was shown to vary by $35 \%$ across the flame so the mean properties are not truly one-dimensional. However, the resulting value of $\bar{w}$ appears to be reasonable since the integral of $\bar{w} d x$, as determined from Fig. 13, agrees with the value of $\rho_{1} S_{T}$ 


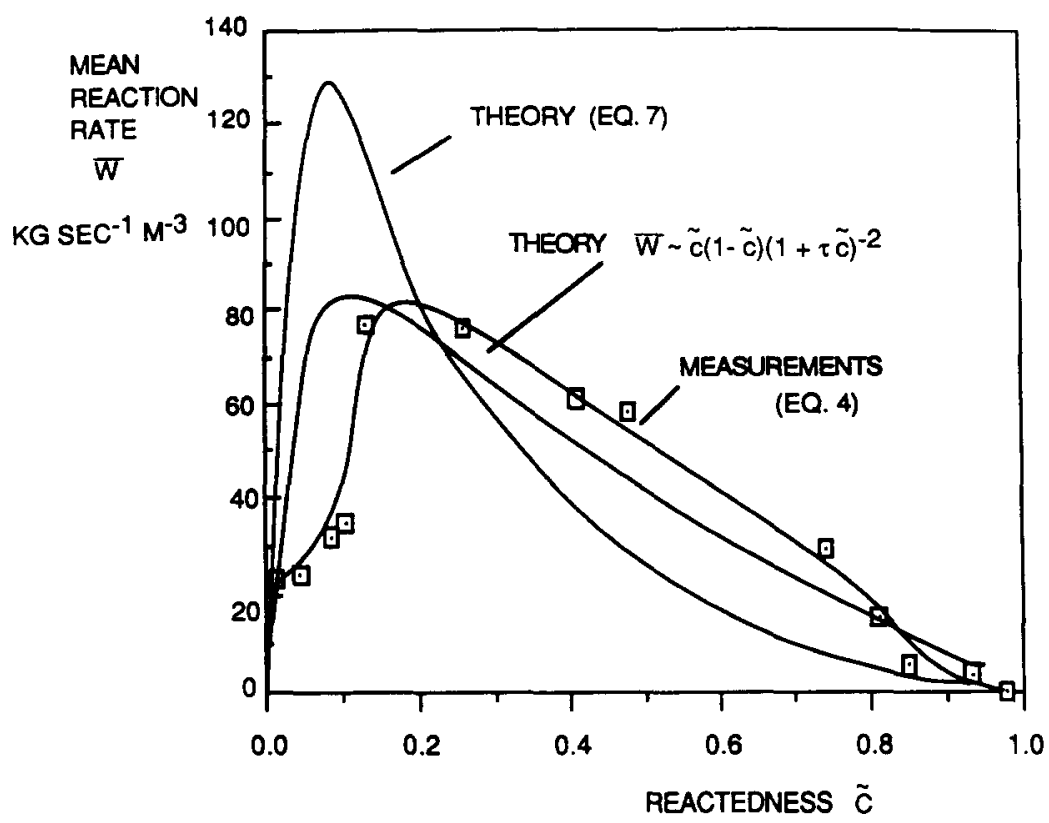

Fig. 13. Mean reaction rates estimated from measurements. Also shown are predictions of BM-L model [26] using empirical values of $\nu_{\mathrm{m}}$ and $\theta$ for present experiment.

to within the $35 \%$ uncertainty of the estimates. The integral of $\bar{w} d x$ represents the mass of products formed per unit cross-sectional area and $\rho_{1} S_{T}$, where $S_{T}$ is the turbulent flame speed, represents the mass of reactants consumed per unit cross-sectional area.

For comparison, Fig. 13 also shows the predicted values of $\bar{w}$ that were obtained from the following modeled relation proposed by Bray, Libby, and Moss [26]:

$\bar{w}=\left[\frac{4 \nu_{\mathrm{m}}(1+\tau) \rho_{1} S_{\mathrm{L}}}{\cos \bar{\theta} \tilde{U}}\right]\left[\frac{\tilde{c}(1-\tilde{c})}{(1+\tau \tilde{c})^{2}}\right]$,

Where $S_{\mathrm{L}}$ is the laminar flame speed. Equation (7) is semiempirical in that experimental values are required for $\nu_{\mathrm{m}}$, which is the average flamelet crossing frequency at the location where $\tilde{c}=0.5$, and for $\bar{\theta}$, which is the average angle between the normal to the flamelet and $U_{1}$. The value of $\nu_{\mathrm{m}}$ was determined from Rayleigh scattering data to be $250 \mathrm{~Hz}$. Schlieren movies showed that $\bar{\theta}$ was approximately $45^{\circ}$ at all locations (except near $\tilde{c}$ $=0$ and 1) because of the semicircular shape of the flamelets and their random sizes and positions.
Predicted values of $\bar{w}$ are shown in Fig. 13; mean reaction rate reaches a maximum in the upstream portion of the flame because the factor $\tilde{U}(1+\tau \tilde{c})^{2}$ in the denominator of Eq. (7) increases rapidly with $\tilde{c}$. In contrast, Yoshida and Gunther [35], who used an ion probe, conclude the maximum value of their estimates of $\bar{w}$ occur in the downstream portion of the flame. A more recent analysis by Libby [36] predicts that mean reaction rate should be proportional only to the second factor in parenthesis in Eq. (7); Fig. 13 shows that such a model more closely agrees with the present results. Figure 13 suggests that further improvements in the modeling of $\bar{w}$ are needed. At present, there is no way to deduce $\nu_{\mathrm{m}}$ or $\bar{\theta}$ from first principles; furthermore, $\nu_{\mathrm{m}}$ varies widely: the value of $\nu_{\mathrm{m}}$ in the present work is one-fifth of that measured by Namazian [18], for reasons discussed in Ref. [12].

\section{F. Additional Comparisons of Measurements to Theory}

The one area in which there is some disagreement between the present measurements and the B-M-L 
model involves the true turbulence associated with products $\left(u_{\mathrm{p}}^{\prime}\right)$. Figure 14 shows that $\left(u_{\mathrm{p}}^{\prime} / U_{\mathrm{p}}\right)$ is found to decrease in the $x$ direction, whereas the opposite trend is predicted by the B-M-L model. The measured values of $u_{\mathrm{p}}^{\prime} / U_{\mathrm{p}}$ are believed to decrease because some of the low speed products that were identified in Fig. 8 mix with higher speed products, causing the velocity PDF of products to become more Gaussian, thereby causing $u_{\mathrm{p}}^{\prime} / U_{\mathrm{p}}$ to decrease. The model predictions of $u_{\mathrm{p}}^{\prime} / U_{\mathrm{p}}^{\mathrm{p}}$ are not in agreement with the measurements for two reasons. First, the model assumes that the velocity PDF of the products is Gaussian (i.e., that no low speed products exist), which does not agree with the data of Fig. 8. Second, the model requires one empirical closure relation in order to relate the fluctuations in the products $\left(u_{\mathrm{p}}^{\prime}\right)$ to other variables. Three possible choices for the required closure equation have been proposed by Libby [6] and by Bray et al. [7]:

$$
\begin{aligned}
i_{\mathrm{p}}-i_{\mathrm{r}}= & -\left(1-K_{\mathrm{p}}\right) I_{0}+\left[(1+\tau)\left(1-K_{\mathrm{r}}\right) I_{\infty}\right. \\
& \left.+\left(1-K_{\mathrm{p}}\right) I_{0}\right] \tilde{\mathcal{c}}
\end{aligned}
$$

$i_{\mathrm{p}}-i_{\mathrm{r}}=-(1-K)(1-2 \tilde{c})\left(\overline{\rho u^{\prime \prime 2}} / \rho U_{1}^{2}\right)$,

$i_{\mathrm{p}}-i_{\mathrm{r}}=K_{3}\left(U_{\mathrm{p}}-U_{\mathrm{r}}\right)^{2}$.

The quantity $\left(i_{\mathrm{p}}-i_{\mathrm{r}}\right)$ is $\left(\overline{u_{\mathrm{p}}^{\prime 2}}-\overline{u_{\mathrm{r}}^{\prime 2}}\right) / U_{1}^{2} ; I_{0}$ is $\overline{\rho u^{\prime 2} / \rho_{1}} U_{1}^{2} ; I_{\infty}$ is $(1+\tau)^{-1} u_{2}^{\prime 2} / U_{1}^{2} ; K_{\mathrm{r}}$ is the limiting value of $\overline{u_{\mathrm{r}}^{\prime 2}} / \overline{u^{\prime 2}}$ as $\tilde{c}$ approaches unity; and $K_{\mathrm{p}}$ is the limiting value of $\overline{u_{\mathrm{p}}^{\prime 2}} / \overline{u^{\prime 2}}$ as $\tilde{c}$ approaches zero. Equation (10) was used for all predictions in the present study.

In order to assess the proposed Eq. (8)-(10), both the right-hand side and the left-hand side of each equation was measured. Figure 15 shows the results obtained for the $\tau=5.5$ flame. Equations (9) and (10) are shown to be invalid in the present flame since the magnitudes and the slopes of the right-hand sides differ from those of the left-hand side. Figure 15 shows that Eq. (8) is significantly better at approximating the correct magnitude and slope of $\left(i_{\mathrm{p}}-i_{\mathrm{r}}\right)$. Libby [6] also has shown that Eq. (8) has a stronger physical basis than Eqs. (9) and (10). However, one conclusion that can be drawn from Fig. 15 is that current closure schemes are not sufficient. It is believed that a more

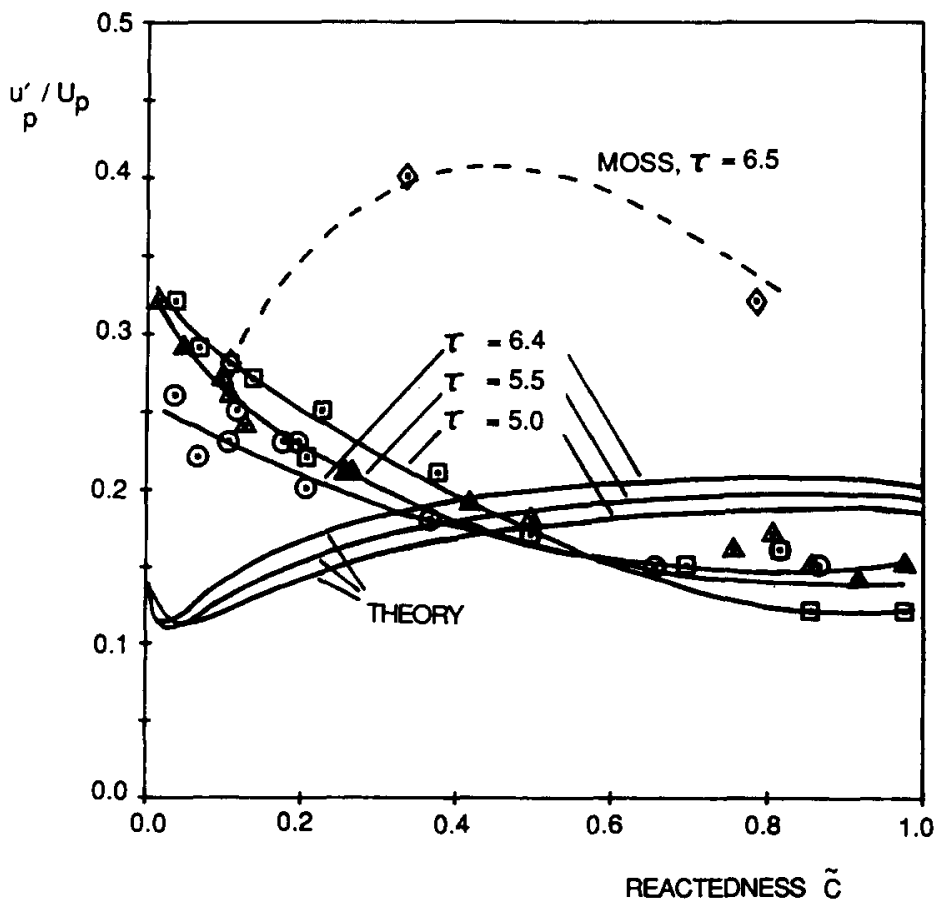

Fig. 14. Effect of heat release on conditioned rms fluctuations of normal velocity of products Data of Moss [13] also are shown. 


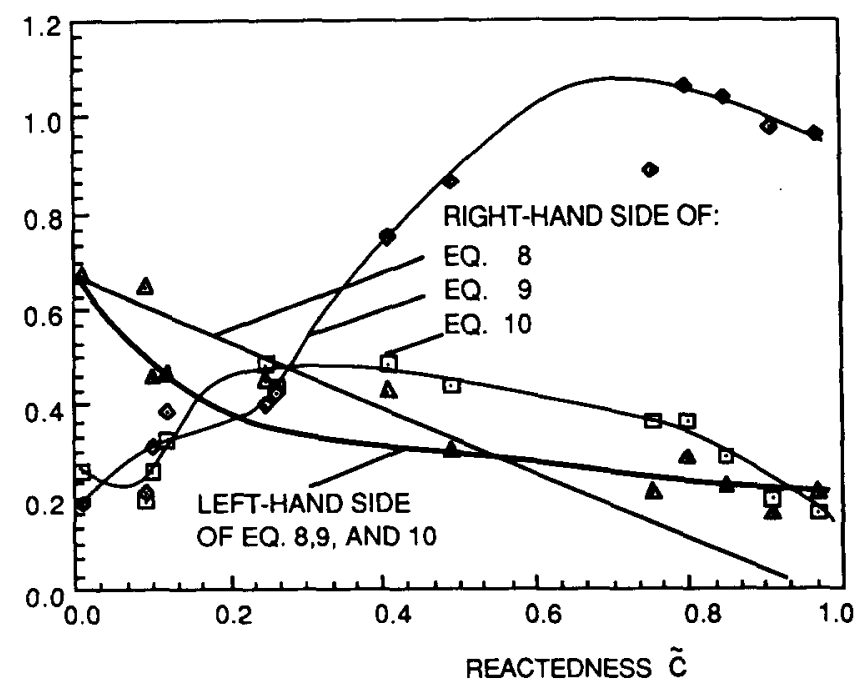

Fig. 15. Assessment of three possible choices as a closure equation for $u_{\mathrm{p}}^{\prime}$.

realistic closure scheme should model the physical mechanism by which flame-generated turbulence arises due to curved flamelets, as was discussed in Subsection D.

In contrast to the above case, there was encouraging agreement between theory and measurements of those quantities which more heavily depend on the apparent turbulence, i.e., the flamelet motion. The production term in the TKE balance was shown in Subsection $\mathrm{C}$ to be one such quantity, since it is proportional to $\overline{\rho^{\prime} u^{\prime}}$. Other examples are $\overline{\rho u^{\prime \prime} c^{\prime \prime}}$ and $\overline{\rho u^{\prime \prime} c^{\prime \prime}}$. The mean flux

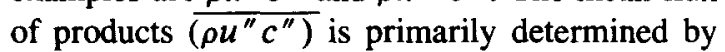
flamelet motion, which leads to the mechanism termed countergradient diffusion [7, 13, 15]. Evidence of countergradient diffusion in the present flame is seen in Fig. 16. The positive

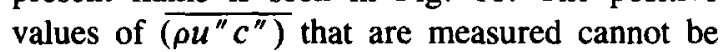
modeled by the gradient diffusion expression $-D_{1}(d \tilde{c} / d x)$ since $d \tilde{c} / d x$ also is positive throughout the flame and $D_{1}$ cannot be negative. The peak value of $\overline{\left(\rho u^{\prime \prime} c^{\prime \prime}\right)}$ is also found to increase with increasing $\tau$, as predicted by the B-M-L model.

The good agreement between measurements and predictions of $\rho u^{\prime \prime} c^{\prime \prime}$ in Fig. 16 is due to the fact that the B-M-L model specifically separates velocities into a bimodal distribution consisting of reactants and products. Countergradient diffusion arises when products travel faster than reactants, (i.e., $\overline{u^{\prime \prime} c^{\prime \prime}}$ is positive); it follows that in order to model countergradient diffusion, a first requirement is to separate the physical properties of reactants from those of products, as is done in the B-M-L model.

It is noted that a laminar flame is required to have sufficient heat transfer in the negative $x$ direction to preheat the reactants to an effective ignition temperature, yet in a turbulent flame there is no evidence from Fig. 16 or similar data in Refs. [7], [11], and [13] that the turbulent heat flux term $\rho C_{\mathrm{p}} u^{\prime} T^{\prime}$ is negative at any location. The positive values of $\overline{u^{\prime} T^{\prime}}$ in Fig. 16 are physically reasonable because the hot products are accelerated and thus have values of $u$ that are larger than average. The turbulent flame propagates, however, because the flamelets within the turbulent flame must meet the above condition, i.e., they must have sufficient molecular heat transfer in the negative $x$ direction and thus most likely they will have portions aligned obliquely to the incident flow. Thus the positive values of $\overline{u^{\prime} T^{\prime}}$ in Fig. 16 do not violate the concept of flame propagation.

The B-M-L model correctly predicts the countergradient trend in the flux of products $\overline{\rho u^{\prime \prime} c^{\prime \prime}}$, but an even more demanding question is whether or not the model adequately predicts the triple correlation terms, especially $\rho u^{\prime \prime 2} c^{\prime \prime}$ which is the flux of the transport quantity $\overline{\rho u^{\prime \prime} c^{\prime \prime}}$. Measurements of the triple correlation $\overline{\rho u^{\prime \prime 2} c^{\prime \prime}}$ are plotted in Fig. 17. Despite some scatter in the measure- 


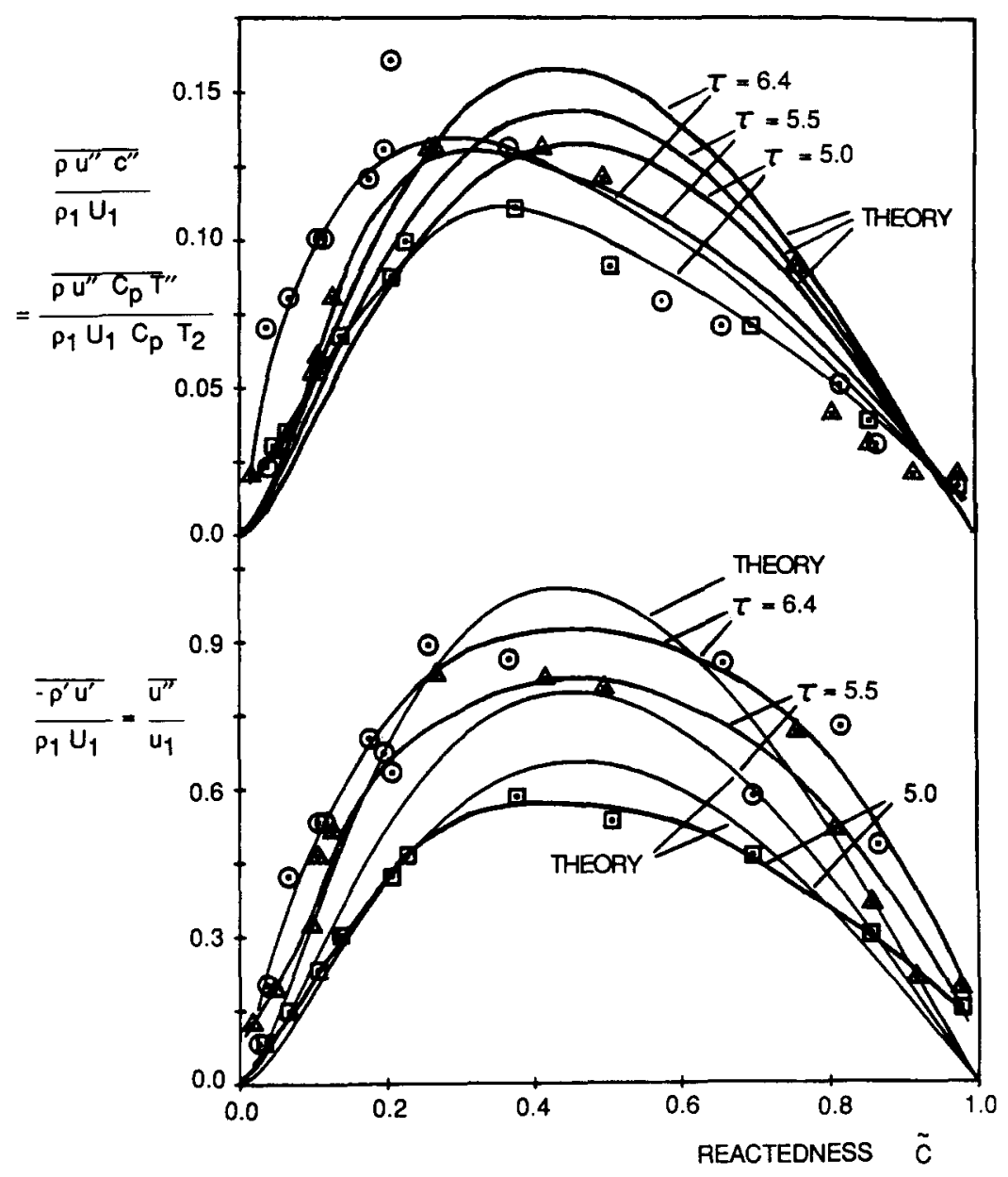

Fig. 16. Turbulent flux of products $\overline{\rho u^{\prime \prime} c^{\prime \prime}}$, heat flux $\overline{\rho u^{\prime \prime} c_{\mathrm{p}} T^{\prime \prime}}$, and mass flux $\overline{\rho^{\prime} u^{\prime}}$, indicating the presence of countergradient diffusion.

ments, it is encouraging that both the measurements and the predictions change sign within the flame and approach zero at the flame brush boundaries.

The measured rms density fluctuations are compared with model predictions in Fig. 18. The measurements are in good agreement with the predicted values; previously, Bill et al. [16] have reported good agreement between measured and predicted values of $\rho^{\prime}$. However, $\rho^{\prime}$ and $\tilde{c}$ are related in the model by two simple and wellestablished relations; thus the good agreement seen in Fig. 18 indicates only that those two simple relations are valid, and does not indicate that the more complex closure relations in the model are valid.
The rate at which flamelets cross a given point within the flame brush has been identified by Bray et al. [26] as an important parameter that determines the mean reaction rate, and thus the flame speed. The Rayleigh scattering signal has a square wave appearance but has an irregular frequency; the flamelet crossing frequency is the number of times per second that the Rayleigh signal changes from either a high value to a low value or vice versa. The B-M-L model predicts that the flamelet crossing frequency $(\nu)$ is given by

$\nu=\nu_{\mathrm{m}} 4 \bar{c}(1-\bar{c})$,

where $\nu_{\mathrm{m}}$ is the maximum value of $\nu$ which is predicted by Eq. (11) to occur at $\bar{c}=0.5$. Measured and predicted values of $\nu / \nu_{\mathrm{m}}$ are plotted 


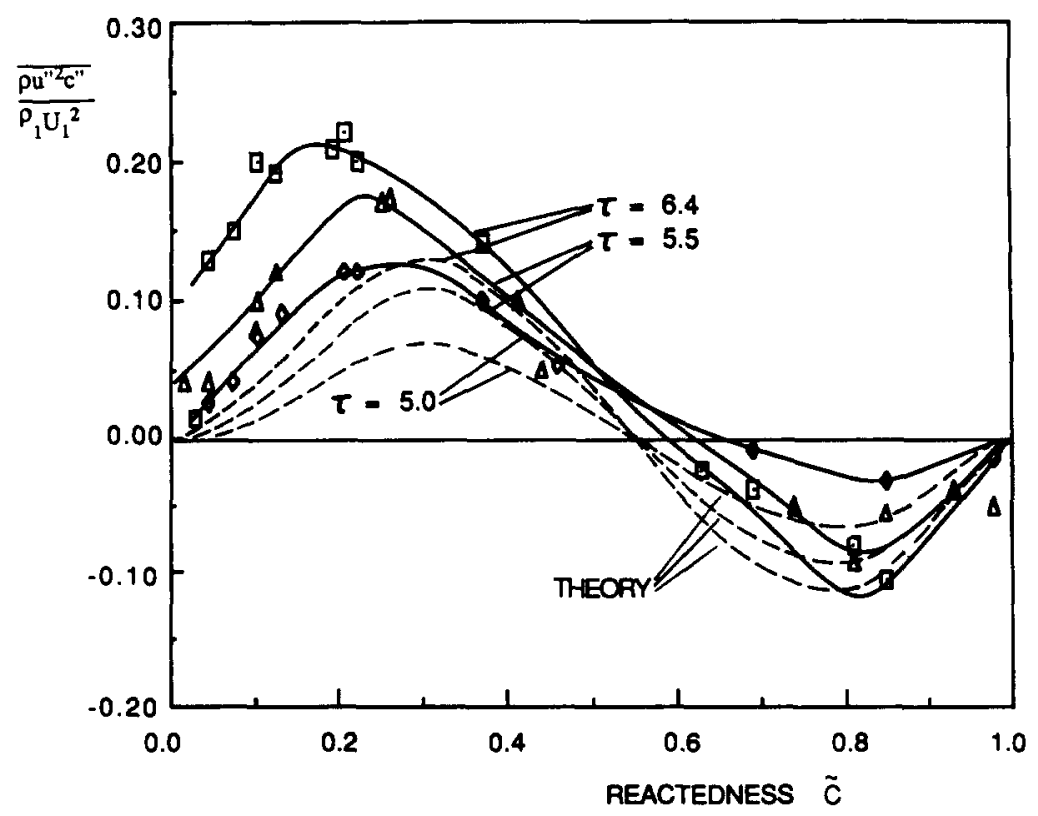

Fig. 17. Measured triple correlation $\overline{\rho u^{\prime 2} c^{\prime \prime}}$ compared with B-M-L theory.

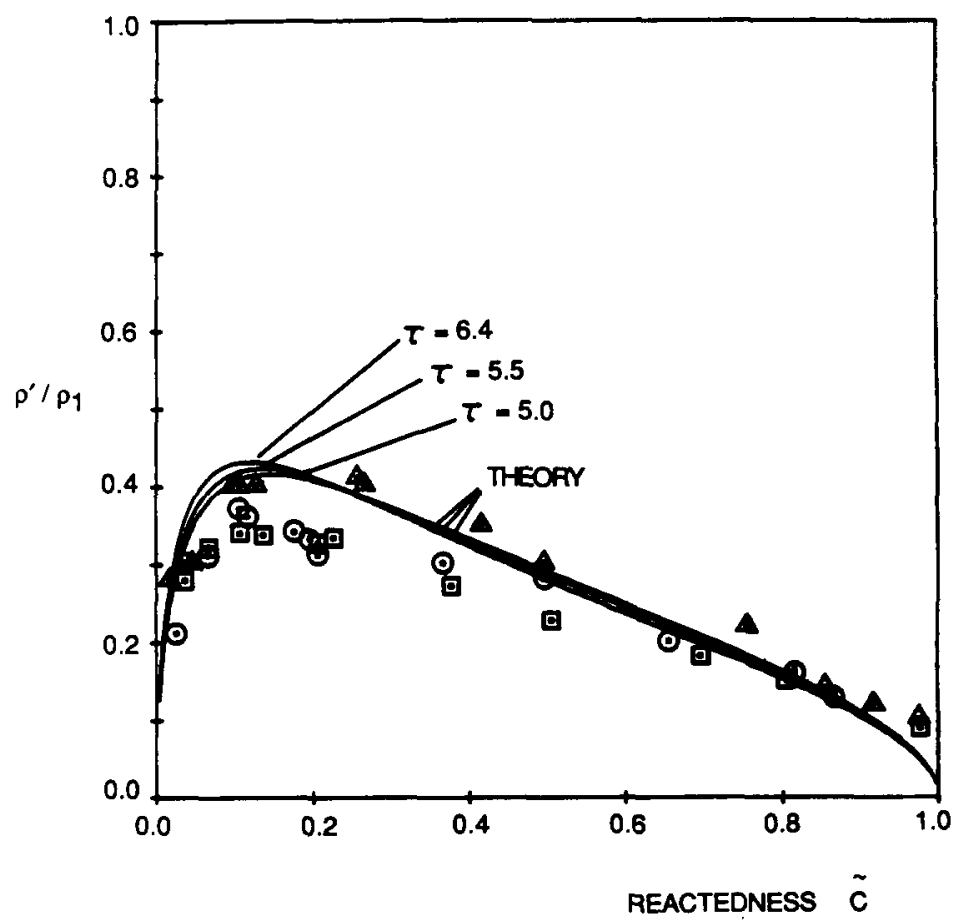

Fig. 18. Rms density fluctuations compared with the predictions of B-M-L model. 
in Fig. 19 and are seen to be in agreement. Similar results were reported by Namazian et al. [18]. The measured value of $\nu_{\mathrm{m}}$ in the present study was 250 $\mathrm{Hz}$ and was found to be independent of heat release.

While only the B-M-L theory has been compared to the present measurements, it is noted that the PDF method of Anand and Pope [37] does predict certain trends that are in better agreement with the present results than the B-M-L predictions. For example, Anand and Pope's model predicts that the velocity PDFs should be skewed, that $\overline{u^{1 / 3}}$ therefore is nonzero [37, Fig. 10], and that transverse velocity fluctuations $\overline{v^{\prime \prime 2}}$ should vary across the flame. Such trends are not predicted by the current B-M-L model but were observed in the present study.

\section{CONCLUSIONS}

1. Four of the six terms in the exact form of the TKE balance were measured; the B-M-L model predicts trends which are in agreement with the measured trends, although the magni- tudes of the measured terms differed by up to $40 \%$ from the predictions. The production term is maximum in the upstream half of the flame, in part because more "apparent turbulence" is produced in this region. The dilatation loss term is maximum in the downstream half of the flame, also partially due to a loss in "apparent turbulence" as flamelet crossings become less frequent. The measured diffusion of TKE was found to occur in a countergradient sense, as the model predicts; it is believed that the diffusion term also is influenced by "apparent turbulence," which, in fact, is the source of the mechanism that is termed countergradient diffusion.

2. Two triple correlations that were measured, $\rho u^{\prime \prime 2} c^{\prime \prime}$ and $\overline{\rho u^{\prime \prime 3}}$, changed from positive to negative values within the flame; the fact that the B-M-L model correctly predicts this sign change is encouraging.

3. The measurements provide direct evidence that some of the "true" turbulence that is produced is due to the curved flamelets that are observed. The flamelets create some unexpected low

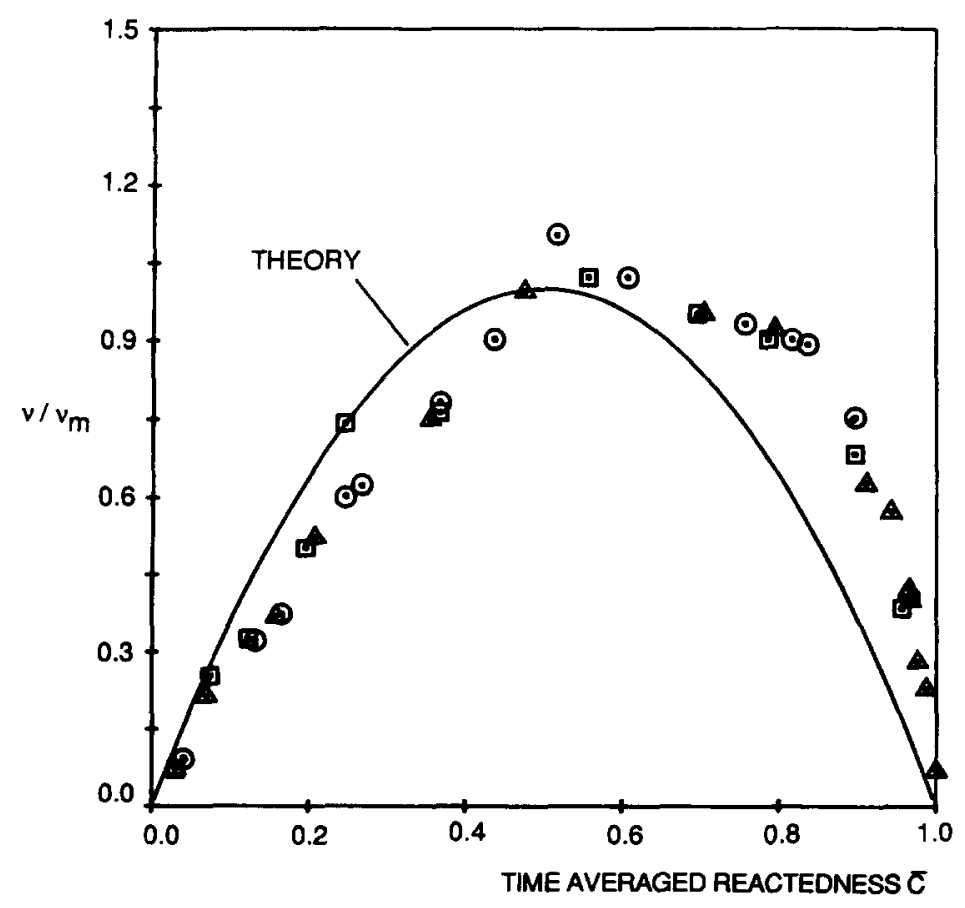

Fig. 19. Flamelet crossing frequency compared with predictions of B-M-L model. 
speed products. This mechanism is not explicitly included in current models.

4. The measurements show that the flame exerts an "upstream influence" that causes the mean velocity and fluctuations of the pure reactants ( $U_{\mathrm{r}}$ and $u_{\mathrm{r}}^{\prime}$ ) to increase before these reactants ever reach a flamelet. The pressure field is the likely source of the upstream influence.

5. The flame-generated turbulence increased monotonically through the flame, whereas the "apparent turbulence" first increases in the upstream portion of the flame, as the velocity PDF evolves from Gaussian to bimodal form, and later decreases in the downstream portion as the velocity PDF evolves back to a Gaussian. The flame causes a net increase in overall turbulence in that $u_{2}^{\prime} / u_{1}^{\prime}$ exceeded unity for all three values of heat release studied. The amount of turbulence generated increases with heat release as predicted by the B-M-L model.

6. One area of disagreement between the present measurements and the B-M-L model involves the turbulence associated with products $u_{\mathrm{p}}^{\prime}$. The velocity PDF of products is not Gaussian; thus $\overline{u_{\mathrm{p}}^{\prime 3}}$ is not zero as assumed by the model, due to the presence of low speed products. The predicted trends in $u_{\mathrm{p}}^{\prime} / U_{\mathrm{p}}$ did not agree with the present measurements. Also, one empirical closure relation that involves $u_{\mathrm{p}}^{\prime}$ in the model does not appear to be universal because it is found to be invalid in the present flame. It appears that further work is needed to model correctly some aspects of the vorticity generation in a flame and how the vorticity may increase the flame sheet area. However, the BM-L model does an excellent job of modeling the apparent turbulence (as well as the many quantities that depend heavily on apparent turbulence) because it separates the reactant and product velocity statistics.

7. Estimates of the mean reaction rate $\bar{w}$ were deduced from the measurements; the model predictions of $\bar{w}$, which are semiempirical in nature, are in fair agreement with the measurements.

This research was supported by National Science Foundation Grant CPE 8213319.

\section{REFERENCES}

1. Lewis, B., and Von Elbe, G., Combustion Flames and Explosions of Gases, Academic Press, 1961.

2. Karlovitz, B., Denniston, D. W., and Wells, F. E., $J$, Chem. Phys. 19:541 (1951).

3. Ballal, D., Proc. Royal Soc. Lond. A376:485 (1979).

4. Ballal, D., AIAA J. 24:1148 (1986)

5. Lee, J. H., Knystautas, R., and Chan, C. K., Twentieth Symposium (International) on Combustion, The Combustion Institute, Pittsburgh, 1984, p. 1663.

6. Libby, P. A., Prog. Energy Combust. Sci. 11:83 (1985).

7. Bray, K. N. C., Libby, P. A., Masuya, G., and Moss, J. B., Comb. Sci. and Tech. 25:127 (1981),

8. Libby, P. A., and Bray, K. N. C., AIAA J. 19:205 (1981).

9. Williams, F. A., and Libby, P. A., AIAA J. 19:261 (1981).

10. Cheng, R. K., Comb. Sci. and Tech. 41:109 (1984).

11. Gulati, A., and Driscoll, J. F., Comb. Sci. and Tech. 48:285 (1986).

12. Gulati, A., and Driscoll, J. F., Twenty-First Symposium (International) on Combustion, The Combustion Institute, Pittsburgh, 1987 (to appear).

13. Moss, J. B., Comb. Sci. and Tech. 22:119 (1980).

14. Shepherd, I. G., and Moss, J. B., AIAA J. 20:566 (1981).

15. Yanagi, T., and Mimura, Y., Eighteenth Symposium (International) on Combustion, The Combustion Institute, Pittsburgh, 1981, p. 1031.

16. Bill, R. G., Jr., Namer, I., Talbot, L., and Robben, F., Combust. Flame 44:277 (1982).

17. Bill, R. G., Jr., Namer, I., Talbot, L., Cheng, R. K., and Robben, F., Combust. Flame 43:229 (1981).

18. Namazian, M., Talbot, L., and Robben, F., Twentieth Symposium (International) on Combustion, The Combustion Institute, Pittsburgh, 1984, p. 411.

19. Gouldin, F. C., and Dandekar, K. V., ALAA J. 22:665 (1984).

20. Dandekar, K. V., and Gouldin, F. C., AIAA J. 20:652 (1982).

21. Masuya, G., and Libby, P. A., AIAA J. 19:1590 (1981).

22. Dibble, R. W., Rambach, G. D., Hollenbach, R. E., and Ringland, J. T., NTIS Report SAND81-8800, 1981.

23. Driscoll, J. F., Schefer, R. W., and Dibble, R. W., Nineteenth Symposium (International) on Combustion, The Combustion Institute, Pittsburgh, 1982, p. 477 .

24. McLaughlin, D. K., and Tiederman, W. G., Phys. Fluids 16:2082 (1973).

25. Doebelin, E. O., Measurement Systems, McGraw-Hill, New York, 1966.

26. Bray, K. N. C., Libby, P. A., and Moss, J. B., Comb. Sci. and Tech. 41:143 (1984).

27. Yoshida, A., and Tsuji, H., Twentieth Symposium (International) on Combustion, The Combustion Institute, Pittsburgh, 1984, p. 445. 
28. Peterson, R., and Emmons, H. W., Phys. Fluids 4:456 (1961).

29. Markstein, G. H., J. Aero. Sci. 18:199 (1951).

30. Strehlow, R. A., Combustion Fundamentals, McGraw-Hill, New York, 1984, Sections 8.6, 10.4, 11.2.

31. Cheng, R. K., and Ng, T. T., Combust. Flame 52:185 (1983).

32. Emmons, H. W., Fundamentals of Gas Dynamics, Vol. III of Princeton Series on High Speed Aerodynamics, Princeton University Press, 1958.

33. Ashurst, W. T., Comb. Sci. and Tech. 52:325 (1987).
34. Schultz, P. H., and Reuss, D. L., Paper 7C1, Central States Section Meeting, The Combustion Institute, NASA Lewis Research Center, Ohio, 1986.

35. Yoshida, A., and Gunther, R., Comb. Sci. and Tech. 26:43 (1981).

36. Libby, P. A., Private communication, 1986.

37. Anand, M. S., and Pope, S. B., Combust. Flame 67:127-142 (1987).

Received 7 January 1987; revised 5 August 1987 